\title{
Impacts of iron on phosphate starvation-induced root hair growth in Arabidopsis
}

\author{
Caiwen Xue ${ }^{1}$, Wenfeng $\mathrm{Li}^{2}$, Ren Fang Shen ${ }^{3}$, and Ping Lan ${ }^{1}$ \\ ${ }^{1}$ Institute of soil science, Chinese academy of sciences \\ ${ }^{2}$ Nanjing Normal University \\ ${ }^{3}$ Institute of soil science, Chinese academy of sciences
}

May 25, 2021

\begin{abstract}
Phosphate is essential for plant growth and development. Root architecture alternations induced by phosphate starvation (-Pi), including primary root and lateral root growth, are mediated by iron (Fe). However, whether and how Fe participates in the -Pi-induced root hair growth (RHG) remains unclear. Here, with morphological, proteomic, and pharmacological analysis, we investigate the impacts of Fe on RHG under -Pi and the underlying mechanisms. We found that -Pi-induced RHG was affected by the local Fe availability. Reduced sensitivity to Fe was found in aux1-7, arf10arf16, and phr1 under -Pi, indicating auxin and phosphate starvation-induced responses were required for the Fe-triggered RHG under -Pi. Fe availability was then found to affect the auxin distribution and expression of phosphate starvation-responsive (PSR) genes. Proteomic analysis indicated vesicle trafficking was affected by Fe under -Pi. With the application of brefeldin A, we found the vesicle trafficking was affected by $\mathrm{Fe}$, and root hairs displayed reduced sensitivity to Fe, indicating the vesicle trafficking is critical for Fe-triggered RHG under -Pi. Our data suggested that Fe is involved in RHG under -Pi by integrating the vesicle trafficking, auxin distribution, and PSR. It further enriches the understanding of the interplay between phosphate and iron on RHG.
\end{abstract}

\section{Introduction}

As an essential component of biomacromolecules, phosphate (Pi) is extensively involved in plants' development and growth, including photosynthesis, energy generation, signaling transduction, and carbohydrate metabolisms (Niu et al. 2013). Phosphate deficiency (-Pi) is a major constrain for agricultural production due to the poor mobility and low bio-availability of phosphate in soil (Mittler 2006).

To overcome the limitation of $\mathrm{Pi}$, robust root architecture remodeling, including altered primary root growth, lateral root formation, crown root angle, and root hairs, would be adopted by plants to enhance the contact with topsoil and facilitate Pi absorption (Huang \& Zhang 2020). The proliferated root hair growth is an early response toward - $\mathrm{Pi}$, compared to the primary roots and lateral roots (Ma et al. 2001). The enhanced root hair growth under -Pi distinctly could significantly facilitate the Pi uptake agricultural system (Bates \& Lynch 2000). Thus, further understanding of -Pi-induced root hair growth is urgent for breeding Pi efficient crops.

Root hairs are single tubular cells derived from specialized epidermal cells (Shibata \& Sugimoto 2019). Root hair growth is a highly coordinated system, including polarized targeting of vesicles, production and accumulation of reactive oxygen species (ROS), proper calcium $\left(\mathrm{Ca}^{2+}\right)$ gradient, and cytoskeleton dynamics (Ovečka et al. 2005; Mendrinna \& Persson 2015; Zeb et al.2020; Zhou et al. 2020). Phosphate deprivationinduced root hair growth has been extensively focused (Shibata \& Sugimoto 2019; Huang \& Zhang 2020). ROOT HAIR DEFECTIVE 6-LIKE 4 (RSL4) is an essential potentiator of root hair growth under -Pi, which is directly regulated by the ROOT HAIR DEFECTIVE 6 (RHD6) (Datta et al. 2015; Yiet al. 2010). 
The PHOSPHATE STARVATION RESPONSE 1 (PHR1), the critical regulator of phosphate starvation responses, is positively involved in root hair growth under phosphate deficiency (Shibata \& Sugimoto 2019; Bustos et al. 2010). Phytohormones, including auxin, ethylene, and jasmonic acid, are essential players in root hair growth (Vissenberg et al. 2020; Parry 2018). Under -Pi, auxin production would be promoted at the root tip, which would be mobilized to the root hair zone by AUXIN RESISTANT 1 (AUX1) and subsequently facilitate the root hair growth (Bhosale et al. 2018; Parry 2018).

Recently, the interconnections between multiple mineral elements have been focused. The phenotypic responses toward -Pi are supposed to be outcomes of the complex interaction between Pi and other nutrients, especially iron (Fe) (Rouached et al. 2010; Briat et al.2015; Müller et al. 2015; Dong et al. 2017). Fe has been noted as a central player in -Pi induced primary root inhibition, mediated by the PHOSPHATE DEFICIENCY RESPONSE2 (PDR2) and LOW PHOSPHATE ROOT 1 (LPR1), as well as the ALUMINUM SENSITIVE 3-3 (ALS3) and SENSITIVE TO AL RHIZOTOXICITY1 (STAR1) (Müller et al. 2015; Donget al. 2017). Fe is also integrated into the -Pi-induced lateral root development in an auxin-dependent way (Rai et al. 2015). The induction of $R S L 4$, a key regulator of root hair growth under -Pi, is dependent on LPR1 and LPR2 (Crombez et al. 2019; Yi et al. 2010), indicating the potential roles of Fe in root hair growth under Pi deprivation. While whether and how Fe is involved in the -Pi induced root hair growth remains unclear.

To decipher the potential role of Fe in -Pi-induced root hair growth, root hairs of Arabidopsis wild type (Col0 ) seedlings were observed treated with different Fe gradients under Pi sufficient and deficient conditions. We found that although the root hair growth is tightly correlated with the local concentration of Fe at both Pi levels. And different mechanisms might be employed in the Fe-triggered root hair growth at two Pi levels, indicated by genetic analysis. Auxin was supposed to be required for the Fe-triggered root hair growth under -Pi, for reduced sensitivity of root hair growth to Fe, had been observed in both auxin-related mutants, aux17 , and arf10arf16, as well as the altered distribution of auxin observed by DR5:GFP. The Fe-triggered root hair growth under -Pi was also dependent onPHR1, and the phosphate starvation responsive (PSR) genes were affected by $\mathrm{Fe}$ availability. Proteomic analysis indicated that the proteins involved in vesicle trafficking and auxin transport and metabolism were altered by Fe, especially under Pi deprivation. The essential role of vesicle trafficking in Fe-dependent root hair growth under -Pi was further indicated by the altered exocytosis affected by Fe and the reduced sensibility of root hair growth to Fe induced by vesicle trafficking antagonist. In all, our work further illustrated the roles of Fe in the -Pi-induced root hair growth, and proved that Fe availability could affect -Pi-induced root hair growth via modulating vesicle trafficking, auxin distribution and phosphate starvation responses. We supposed that Fe is integrated into the -Pi induced root hair growth via altering vesicle trafficking, which might lead to the altered auxin distribution and PSR genes, and consequently regulating the root hair growth.

\section{Materials and methods}

\section{Plant Growth Conditions and Treatments}

The ecotype Columbia-0 of Arabidopsis thaliana was used as the wild type (WT) in this study. After being surface sterilized and vernalized at $4^{\circ} \mathrm{C}$ for $2 \mathrm{~d}$, seeds were germinated on the Petri plates in a growth chamber with a 16-h-light/8-h-dark photoperiod at $22{ }^{\circ} \mathrm{C}, 70 \%$ humidity. Different treatments were performed based on the modified ES media (Estelle and Somerville, 1987). The $\mathrm{Pi}$ sufficient $(+\mathrm{Pi})$ media was prepared as described (Huang et al., 2017), and -Pi medium was prepared by replacing $\mathrm{KH}_{2} \mathrm{PO}_{4}$ with $\mathrm{KCl}$. $\mathrm{Fe}(\mathrm{III})$-EDTA, sterilized by filtration, was added into the $+\mathrm{Pi} /-\mathrm{Pi}$ media after sterilization.

\section{Observation and Measurement of Root Hair}

Seedlings were grown on ES media for $5 \mathrm{~d}$ and were transferred for $9 \mathrm{~d}$ treatment. Root hairs of the 14-d-old seedlings were observed with a Stemi-305 microscope (Zeiss). For each treatment, at least three biological repeats were performed. Root hairs within the scope of $4-6 \mathrm{~mm}$ from the apex were measured and calculated. Root hair length was measured by Image J (1.4.3.67), and data were analyzed by Sigma Plot (12.5). For brefeldin A (BFA) treatment, seedlings grown on ES media for $5 \mathrm{~d}$ and were transferred to treatment media 
applied BFA. $500 \mathrm{nM}$ BFA and $15 \mu \mathrm{M}$ BFA were used, and root hairs of seedlings treated for $3 \mathrm{~d}, 5 \mathrm{~d}$, and $7 \mathrm{~d}$ were observed.

Split-Root experiment

Split-Root experiment has been performed as described with slight modification (Saiz-Fernández et al., 2021). Partial de-rooting had been performed on the main roots of 4-day-old seedlings grown on +Pi media to induced secondary roots. And after three days, seedlings were transferred for treatments, with two induced secondary roots treated with different Fe gradients. Root hairs of each root were observed after $7 \mathrm{~d}$ treatment.

RNA Extraction and Quantitative Real-time PCR ( qRT-PCR)

TRIzol reagent (Invitrogen) was used for total RNA extraction, and the reverse transcription was conducted with $1 \mu \mathrm{g}$ total RNA with reverse transcription kit (TaKaRa, with gDNA Eraser, Cat\#RP047A). Quantitative real-time PCR was performed with TB Green Perfect mix (TaKaRa, Cat\# RR420A) by PIKOREAL 96 RealTime PCR System (Thermo). TUA3(tubulin alpha-3, At5g19770 ) was used as the internal reference gene, and primers used were listed in the supplementary Table S1.

Observation of Reactive Oxygen Species (ROS) Activity and Fluorescent Proteins

The fluorescent dye, 2',7'-dichlorodihydrofluorescein diacetate $\left(\mathrm{H}_{2} \mathrm{DCF}-\mathrm{DA}\right)$, was used to observe ROS activity with slight modification (Mangano et al., 2017). Roots of 10-d-old seedlings (grown on +Pi media for $5 \mathrm{~d}$ and transferred for 5-d-treatment) were incubated with $50 \mu \mathrm{M} \mathrm{H}_{2} \mathrm{DCF}$-DA for $10 \mathrm{~min}$ and washed with washing buffer $\left(0.1 \mathrm{mM} \mathrm{KCl}\right.$ and $\left.0.1 \mathrm{mM} \mathrm{CaCl}_{2}, \mathrm{pH} 6.0\right)$ subsequently. Images were taken by the Zeiss (LSM 710) confocal microscope, the ROS levels of roots were quantified by Image J (version 1.4.3.67). The fluorescent signal of the DR5:GFP was observed by the Zeiss (LSM 710) confocal microscope. The fluorescent signal was estimated by Image $\mathrm{J}$ (version 1.4.3.67) and the signal range scanning was performed by ZEN 2.6 (blue edition).

Vesicle Trafficking Staining Assay

The lipophilic styryl dye, FM4-64, was used for tracing the vesicle trafficking as described (Deng et al. , $2015)$ with mild modification. Roots of 10-d-old seedlings were stained with FM-4-64 (5 $\mu \mathrm{g} / \mathrm{ml})$ for $10 \mathrm{~min}$ and were washed for treatment of BFA $(50 \mu \mathrm{M})$ for $15 \mathrm{~h}$. The effects of BFA on vesicle trafficking were observed after treating with BFA for $15 \mathrm{~h}$ and after removing the BFA for another $15 \mathrm{~h}$. The Zeiss (LSM 710) confocal microscope was used for observation, and the average sizes of the BFA bodies were measured by Image J (version 1.4.3.67),

Proteomic preparation and label-free nanoLC-MS/MA analysis

Protein extraction, digestion, and desalination were performed as previously described in our previous work (Lan et al. 2012; Yanet al. 2020).\{Lan, 2012 \#178;Yan, 2020 \#179\} Roots of 13-d-old seedlings (10-d-old seedlings transferred for $3 \mathrm{~d}$ treatment) were collected. And three biological repeats of each treatment were performed. For label-free nanoLC-MS/MA analysis, the mass spectrometry method was slightly modified. The separation of peptides was performed at a flow rate of $0.3 \mu \mathrm{L} / \mathrm{min}$. The total mass scan lasted $90 \mathrm{~min}$ by adjusting the gradient of buffer B $(0.1 \%(\mathrm{v} / \mathrm{v})$ formic acid, $99.9 \% \mathrm{ACN}(\mathrm{v} / \mathrm{v}))$. During the first $85 \mathrm{~min}$, $3 \%$ buffer B was used. Buffer B was increased to $7 \%$ within 15 min and increased to $35 \%$ within 60 min subsequently. Finally, buffer B was increased from $35 \%$ to $80 \%$ within 1 min and maintained at $80 \%$ buffer B for 5 min. At $76 \mathrm{~min}$, the buffer B was decreased from $80 \%$ to $3 \%$ within $30 \mathrm{~s}$ and kept with $3 \%$ buffer B for $135 \mathrm{~min}$. Two technique repeats were performed for each biological replicate.

Database Search and Statistical Analysis

The identification and quantification of proteins were carried out by the Proteome Discoverer software (version 2.2, Thermo Scientific). Searches were conducted against the protein database (Arabidopsis thaliana (TAIR TaxID =3072)) and the decoy database. The decoy database, containing the randomized sequences of the original database, was acquired with the algorithm SEQUEST, which is integrated into the software. 
Trypsin digestion was selected, and two missed cleavages were allowed $10 \mathrm{ppm}$ and $0.02 \mathrm{Da}$ were set as the peptide tolerance and the fragment mass tolerance, respectively. Proteins with peptide confidence higher than $95 \%(\mathrm{p}<0.05)$ were defined as "Master protein." The Protein FDR Confidence was assigned as "High" Proteins, found in at least one treatment and the Exp q-value was less than 0.01, were assigned as the "High" confidential proteins. Proteins with the Exp q-value between 0.05 and 0.01 were assigned as "Medium" confidential proteins.

\section{Bioinformatic analysis}

Proteins with Exp q-value $<0.05$ were selected for further analysis. Proteins with fold change $>=1.5$, and the adj.P-Value $<0.05$ were considered as the 'Differentially Accumulated Proteins' (DAPs). Gene Ontology (GO) annotation and GO enrichment analysis were performed with ClueGo (version 2.5.7) and CluePedia (version 1.5.7) embedded in Cytoscape (version 3.7.2). KEGG analysis was performed by Metascape (https://metascapeorg) (Zhouet al. , 2019), with mini overlap=3, min richment=1.5, and p-Value $<0.05$. Heat maps of DAPs were drawn by TBtools (version 1.046).

\section{Results}

Root hair growth is dependent on the Fe availability under Pi sufficient and deficient condition

To decipher whether Fe participates in the -Pi-induced root hair growth, we observed the root hair growth of the seedlings treated with various Fe gradients $(-\mathrm{Pi}+0 / 10 / 20 / 40 / 100(\mu \mathrm{M}) \mathrm{Fe})$ under $-\mathrm{Pi}$. Results showed that, under $-\mathrm{Pi}$, the root hair growth was tightly correlated with the Fe availability. The root hair length was positively correlated with the Fe concentration within the range of 0-20 $\mu \mathrm{M}$. While the root hair growth started to be inhibited by $\mathrm{Fe}$ as the Fe concentration within was higher than $20 \mu \mathrm{M}$. At $20 \mu \mathrm{M} \mathrm{Fe}$, the longest root hair was observed. Root hair length under $20 \mu \mathrm{M}$ Fe was around 2-fold longer than that under $0 \mu \mathrm{M}$ $\mathrm{Fe}$ and about 3-fold longer than that under $100 \mu \mathrm{M}$ Fe (Fig. 1B, C). The root hair density also displayed apparent dependence on Fe under -Pi (Fig. 1D). Within the range of 0-20 $\mu \mathrm{M} \mathrm{Fe}$, root hair density was positively correlated with $\mathrm{Fe}$ concentration. The root hair density started to decrease as higher Fe (III) concentration was applied (20-100 $\mu \mathrm{M})($ Fig. 1D).

To confirm whether the Fe-triggered root hair growth is limited to $-\mathrm{Pi}$, root hair growth under different Fe gradients $(+\mathrm{Pi}+0 / 40 / 150 / 350 / 500(\mu \mathrm{M}) \mathrm{Fe})$ was also observed under sufficient Pi. Fe-dependent root hair growth had also been found under $+\mathrm{Pi}$. Under Pi sufficient condition, root hair length was positively affected by Fe within the range of 0-350 $\mu \mathrm{M}$. While, as the increase of Fe concentration, root hair length was negatively affected by $\mathrm{Fe}(350-500 \mu \mathrm{M})$. The longest root hair was observed at $350 \mu \mathrm{M} \mathrm{Fe}$, that the root hair length was around 2-fold longer than that under either $0 \mu \mathrm{M}$ or $500 \mu \mathrm{M} \mathrm{Fe}$ (Fig. 1A, C). Unlike -Pi, the root hair density was positively correlated with the Fe concentration under $+\mathrm{Pi}$ (Fig. 1D). These results showed that the Fe availability is integrated with the root hair growth under Pi deficient and Pi sufficient conditions. And so far, the Fe-triggered root hair growth seems to be independent of Pi levels. For further analysis, we selected three Fe gradients at each Pi levels, respectively, including $0 \mu \mathrm{M}, 350 \mu \mathrm{M}, 500 \mu \mathrm{M}$ under $+\mathrm{Pi}$ and $0 \mu \mathrm{M}, 20 \mu \mathrm{M}, 100 \mu \mathrm{M}$ under -Pi.

Fe-triggered root hair growth is induced by local Fe availability at both Pi levels

To distinguish whether it is the systematic or the local Fe signals that trigger the root hair growth at both Pi levels, we perform the split-root analysis (Fig. 2A). For each Pi level, three Fe gradients were randomly paired. Results showed that different root hairs were observed in two separated roots of the same plants treated with different $\mathrm{Fe}$ gradients (Fig. 2B). Under $+\mathrm{Pi}$, root hairs at $350 \mu \mathrm{M} F$ were denser and longer than $0 \mu \mathrm{M}$ Fe (Fig. 2C). Root hairs between 0 and $500 \mu \mathrm{M}$ Fe showed no significant differences in root hair length, but denser root hairs were observed at $500 \mu \mathrm{M}$ Fe (Fig 2B). Root hairs at $350 \mu \mathrm{M}$ Fe were longer than $0 \mu \mathrm{M}$ Fe (Fig. 2D). Under -Pi, seedlings at $20 \mu \mathrm{M}$ Fe showed denser and longer root hairs than 0 and $100 \mu \mathrm{M}$ Fe (Fig. 2F, H). At $0 \mu \mathrm{M} \mathrm{Fe}$, seedlings showed denser and longer root hairs than $100 \mu \mathrm{M} \mathrm{Fe}$ (Fig. $2 \mathrm{G}$ ). The differences of root hair length and root hair density between each Fe gradients observed in the split root system were consistent with that in the complete roots of seedlings treated with different Fe gradients. 
These results indicated that it is the local Fe availability that triggers the root hair growth at both Pi levels.

Fe-dependent root hair growth requires RHD6/RSL1 and RSL4/RSL2 at both Pi levels

In Arabidopsis , RHD6 and its homolog RHD6-Like 1(RSL1) are critical regulators of root hair development (Fenget al. 2017). They directly enhance the transcription of RSL4 and RSL2, which are essential for root hair growth (Shibata \& Sugimoto 2019; Yi et al. 2010). To confirm the dominance of these key root hair regulators in Fe-dependent root hair growth. We assessed the root hair growth of rhd6rsl1 andrsl4rsl2 treated with different Fe gradients at both Pi levels. Both rhd6rsl1 and rsl4rsl2 are noted to be hairless under normal conditions (Feng et al. 2017). And we found bothrhd6rsl1 and rsl4rsl2 remained hairless despite the Fe concentration under either Pi sufficient or Pi deficient condition (Fig. 2A, B, C). These results indicated that the Fe-triggered root hair growth is mediated by RHD6/RSL1 and RSL4/RSL2, and Fe might act upstream of these two regulators.

$R S L 4$ is supposed to be at the bottom of the regulatory network of root hair growth regulating the transcription of series root hair-related genes (Vijayakumar et al. 2016; Mangano et al. 2017; Shibata \& Sugimoto 2019). As the Fe-dependent root hair growth is supposed to be mediated by the RHD6/RSL1 -RSL4/RSL2 pathway, how the $\mathrm{Fe}$ availability affects the downstream of this pathway raised our attention. To answer this, we detected the expressions of several root hair-related genes, including RSL4 and other six genes (MRH6 (At2g03720),RHS13 (At4g02270), RHS19 (At5g67400), RNS1(At2g02990), EXPA7 (At1g12560), and EXP8 (At2g40610), acting at downstream of RSL4 (Vijayakumar et al. 2016; Yiet al. 2010). Under Pi sufficient condition, transcription of few genes, including EXPA7 and EXP8, were slightly enhanced at 350 $\mu \mathrm{M}$ compared to the 0 and $500 \mu \mathrm{M}$ Fe. The expressions of RSL4 and MRH6 were higher than that at $0 \mu \mathrm{M}$ Fe (Fig. 2H). More robust effects of $\mathrm{Fe}$ on the expressions of these genes were observed under - $\mathrm{Pi}$. Under - $\mathrm{Pi}$, the expression of $R S L 4$ was significantly enhanced with the application of $\mathrm{Fe}$, and other root hair-related genes showed the strongest induction at $20 \mu \mathrm{M}$ Fe (Fig. $2 \mathrm{H}$ ). These results implied that RHD6/RSL1 and $R S L 4 / R S L 2$ are necessary for Fe-triggered root hair growth. And robust effects of Fe availability on the transcription of $R S L 4$ and its downstream genes under -Pi hinted that different processes might be integrated into the Fe-dependent root hair growth under different Pi levels.

Fe-triggered root hair growth at two Pi levels is differentially altered in hormone-related mutants

To uncover the roles of auxin and ethylene in the Fe-dependent root hair growth under phosphate deficient and sufficient conditions, we performed morphological analysis on auxin/ethylene-related Arabidopsismutants. Two auxin-related mutants, aux1-7, an auxin influx mutant (Maher \& Martindale 1980; Rahman et al. 2002), andarf10arf16 , a double mutant of auxin response factors (Wanget al. 2005), were used. For ethylene, eil3eil1 , a double-mutant of two essential ethylene response regulators (Dolgikhet al. 2019) was selected. Under Pi sufficient condition, responses of root hair length in aux1-7, arf10arf16, andein3eil1 to Fe displayed no significant differences compared to the WT (Fig. 3; Table 1), indicating the auxin and ethylene are not necessary for the Fe-triggered root hair growth under $+\mathrm{Pi}$. Under Pi deprivation, the ein3eil1 showed no notable significance compared to the WT (Fig. 3A, B, G, H; Table 1). In comparison, root hair growth of two auxin-related mutants showed reduced sensitivity toward Fe availability. The relative root hair length of aux1-7 between 20 and $100 \mu \mathrm{M}$ Fe was about $30 \%$ less than that of the WT (Fig. 3A, B, $\mathrm{C}, \mathrm{D}$; Table 1). Root hairs of arf10arf16 showed the most weakened responses to Fe under -Pi. The relative root hair length ofarf10arf16 between 20 and $0 \mu \mathrm{M}$ Fe was $70 \%$ less than that of WT and was $44 \%$ less than that of the WT between 20 and $100 \mu \mathrm{M}$ Fe (Fig. 3A, B, E, F; Table 1). According to the results, auxin and ethylene are probably not necessary for the Fe-mediated root hairs growth under Pi sufficient condition. In contrast, the auxin is essential for the Fe-mediated root hair growth under $\mathrm{Pi}$ deprivation. These results indicated a unique process might be employed in Fe-triggered root hair growth under -Pi, although similar root hair responses to Fe availability had been observed at different Pi levels.

PHR1 is essential for the Fe-triggered root hair growth under Pi deprivation

The above results suggested the potential unique mechanisms underlying the Fe-triggered root hair growth under - $\mathrm{Pi}$. We then set to uncover how the Fe availability is integrated into the -Pi-induced root hair 
growth.PHR1, an essential regulator of Pi starvation responses, is positively involved in root hair growth under -Pi (Shibata \& Sugimoto 2019) and is extensively involved in the interplay of multiple nutrients (Briat et al. 2015). Here, we observed the root hair of $p h r 1$ treated with different Fe gradients under both Pi levels. Results showed that, under $+\mathrm{Pi}$, both the root hair length of $p h r 1$ showed similar responses to Fe compared to the WT (Fig. 4A, B, C, D; Table 1). While under -Pi, the root hair length of phr1 showed distinct reduced sensitivity to Fe compared to the WT. The relative root hair length of which was around $50 \%$ less than that of the WT between 20 and $0 \mu \mathrm{M} \mathrm{Fe}$, as well as 20 and $100 \mu \mathrm{M} \mathrm{Fe}$ (Table 1). The reduced sensitivity of rot hair growth to Fe under -Pi in phr1 indicated that the Fe affects -Pi-induced root hair growth probably via

PHR1.

PHR1 is an essential regulator of $\mathrm{Pi}$ starvation responses. Thus, we wondered whether the availability of $\mathrm{Fe}$ affects the $\mathrm{Pi}$ starvation responses regulated by PHR1. We then detected the relative expression of PHR1 and several phosphate starvation-induced (PSI) genes regulated by PHR1, including miR399 (At2g34208),RNS1 (At2g02990), SPX3 (At2g45130), PHT1;4(At2g38940), IPS1 (At3g09922) and ACP5 (At3g17790). Results showed that all selected genes, except PHR1, were significantly induced by -Pi. Under $+\mathrm{Pi}$, the relative expressions of PSI genes and PHR1 showed no noticeable differences among different Fe gradients (Fig. 4E). Under -Pi, PHR1 and most PSI genes displayed higher expressions at $20 \mu \mathrm{M}$ Fe than 0 and $100 \mu \mathrm{M} \mathrm{Fe}$ (Fig. 3E). These indicated that the Fe availability could affect the phosphate starvation responses, and PHR1 is involved in the Fe-triggered root hair growth under -Pi.

ROS activity is differentially affected by Fe availability

The iron (Fe) homeostasis is integrated with the production of ROS (Reytet al. 2014), which is a crucial player that determines the cell shape of root hairs (Carol \& Dolan 2006). To answer whether the Fe-triggered root hair growth is correlated with the ROS activity, we used the $\mathrm{H}_{2}$ DCF-DA to observe the ROS activity (Fig. $6 \mathrm{~A}, \mathrm{~B}$ ). When $\mathrm{Pi}$ is sufficient, the ROS activity is positively correlated with the Fe concentration, which is partially consistent with root hair growth (Fig. 6C). Under -Pi, ROS activity was significantly increased compared to $+\mathrm{Pi}$. The highest ROS activity was found at $20 \mu \mathrm{M} \mathrm{Fe}$, and the ROS activity dramatically decreased at $100 \mu \mathrm{M}$ Fe (Fig. 6D), which was consistent with the root hair growth. These results showed that $\mathrm{Fe}$ availability regulates the root hair growth via affecting the ROS activity.

Proteins involved in vesicle trafficking are differentially accumulated in different Fe treatments, notably under Pi deficiency

To investigated the underlying mechanisms of Fe-triggered root hair growth, we performed proteomic research following the procedure (Fig. S1). In total, 3403 proteins were identified, among which 3087 proteins were high confidential proteins, with the Exp q-value less than 0.01, and 316 proteins were medium confidential proteins, with the Exp q-value between 0.05 and 0.01 (Table S3). DAPs of seven groups, $+\mathrm{Pi}+0$ Vs.350, +Pi+350Vs.500, $+\mathrm{Pi}+500 \mathrm{Vs} .0,-\mathrm{Pi}+0$ Vs.20, -Pi+20Vs.100, -Pi+100Vs.0, and +Pi+0Vs.$\mathrm{Pi}+0$, were screened with the threshold of fold change $>=1.5$, and the adjusted P-Value $<0.05$. The proteins detected in each treatment and DAPs were listed in supplementary figures (Fig. S2; Fig. S3; Table S4).

To dissect the functional proteins involved in the Fe-triggered root hair growth, DAPs of four groups, including $+\mathrm{Pi}+350 \mathrm{Vs} .0,-\mathrm{Pi}+20 \mathrm{Vs} .0,+\mathrm{Pi}+350 \mathrm{Vs} .500,-\mathrm{Pi}+20 \mathrm{Vs} .100$, were selected for further analysis. GO enrichment analysis were performed with the DAPs, and the enriched GO terms were further clustered into different groups. Among which, Go terms with the lowest P-Value of each group were listed out (Fig. 7). Under $+\mathrm{Pi}$, processes including protein transport and Golgi vesicle transport were enriched by DAPs of $350 \mathrm{Vs} .0$ and 350Vs.500. Process of response to hydrogen peroxide was specifically enriched by DAPs of $350 \mathrm{Vs} .0$, and processes of root epidermal cell differentiation and polysaccharide biosynthetic process were specifically enriched by DAPs of 350Vs.500 (Fig. 7A). Under -Pi, processes including Golgi vesicle transport and root epidermal cell differentiation were enriched by DAPs of 20Vs.0 and 20Vs.100. Processes of protein transport, auxin metabolic processes were specifically enriched by DAPs of 20Vs.0. Processes of auxin transport and exocytosis were specifically enriched by DAPs of 20Vs.100 (Fig. 7B). Abundances of the 
DAPs involved in these processes were analyzed. Results showed that processes of Golgi vesicle transport, auxin transport and metabolism, and root epidermal cell differentiation showed stronger disturbance induced by Fe under -Pi. Most proteins displayed the strongest induction at $20 \mu \mathrm{M}$ Fe compared to the other two gradients (Fig. 7C, E, F, G). Process of polysaccharide biosynthetic displayed stronger response to Fe under $\mathrm{Pi}$ sufficient condition. Most proteins were robust induced at $350 \mu \mathrm{M}$ Fe and were repressed at $500 \mu \mathrm{M} \mathrm{Fe}$ under $+\mathrm{Pi}$ (Fig. 7B).

KEGG enrichment analysis was also performed. Enriched KEGG pathways filtered with P-Value $<0.05$ were listed (Fig. S4). Processes including RNA transport, SNARE interaction in vesicular transport, and spliceosome were enriched in all groups (Fig. S4). Soluble NSF attachment protein receptors (SNAREs), located on the vesicles and target membranes, are essential for cell expansion and root hair growth (Ichikawa et al. , 2014; Larson et al. , 2014; Sogawaet al. , 2019). The KEGG enrichment results indicated that the SNARE interaction in vesicular transport was strongly affected by Fe. To further understanding the effects of Fe on this pathway, abundances of the enriched proteins had been marked through the process (Fig. 6). Results showed that, under Pi sufficient condition, more proteins showed the highest abundances at $350 \mu \mathrm{M}$ compared to the 0 and $500 \mu \mathrm{M}$ Fe (Fig. 8). Under -Pi, more proteins were affected compared to the Pi sufficient condition. That 10/15 proteins showed the highest abundances at the $20 \mu \mathrm{M} \mathrm{Fe}$. ARF3 and VAMP711 were sensitive to $100 \mu \mathrm{M} F$, that the abundances of which were notably decreased. HAP13, VAMP713, EHD1, and VTI1, were positively correlated with the Fe(III) application, that the abundances of which were increased as the Fe (III) supplied under -Pi (Fig. 8). In conclusion, the process of polysaccharide biosynthetic process displayed a stronger response to $\mathrm{Fe}$ under $+\mathrm{Pi}$. Processes of vesicle trafficking, auxin transport, and auxin metabolism were preferentially affected by Fe under -Pi.

Vesicle trafficking and auxin distribution is affected by Fe under Pi deficiency

Based on the proteomic analysis, we further confirmed the influence of $\mathrm{Fe}$ on vesicle trafficking and auxin transport. For observation of vesicle trafficking, the vesicle trafficking inhibitor, brefeldin A (BFA), and the lipophilic styryl dye, FM4-64, were used (Fig. 9). FM4-64 is used for dying of the bio-membrane, and with BFA treatment, the vesicle trafficking would be inhibited, and visible BFA bodies would be formed (Deng et al. 2015; Fig. 9a, b, f). Results showed that, after $1.5 \mathrm{~h}$ BFA treatment, the BFA bodies were formed, indicating the inhibition of vesicle trafficking (Fig. 9A, F). And the BFA bodies became smaller after removing the BFA for another $1.5 \mathrm{~h}$, implying the recovery of the inhibition on vesicle trafficking (Fig. 9C, G). Under $+\mathrm{Pi}$, no significant differences had been observed among different Fe gradients after either 1.5 $\mathrm{h}$ BFA treatment or removing BFA for $1.5 \mathrm{~h}$ (Fig. 9D, E). In contrast, under -Pi, seedlings treated with 20 $\mu \mathrm{M}$ Fe showed significant insensitivity to the BFA treatment. BFA bodies formed after $1.5 \mathrm{~h}$ BFA treatment or removing BFA for $1.5 \mathrm{~h}$, in seedlings treated with $20 \mu \mathrm{M} \mathrm{Fe}$, were notably smaller than that of the other two gradients $(0$ and $100 \mu \mathrm{M})($ Fig. 9H, I). These indicated that the vesicle trafficking was strongly affected by Fe under -Pi. Thus we suggested that Fe availability affects -Pi-induced root hair growth probably via modulating vesicle trafficking.

The genetic analysis of auxin-related mutants and proteomic research showed that auxin is required for the Fe-triggered root hair growth under -Pi, probably due to the altered transport of auxin. Thus, we used the transgenic material carrying DR5:GFP, to observe the distribution of auxin (Fig. 10). The linear fluorescence scanning was performed, and we found that, under Pi sufficient condition, the fluorescence length displayed no significant differences across different Fe gradients (Fig. $10 \mathrm{~A}, \mathrm{~B}$ ). Under -Pi, the longest fluorescence length was observed at $20 \mu \mathrm{M} \mathrm{Fe}$, which was notably longer than that at $0 \mu \mathrm{M} F$ and was slightly longer than that at $100 \mu \mathrm{M}$ Fe (Fig. $10 \mathrm{~A}, \mathrm{C}$ ), which was consistent with the root hair growth. These results showed that the auxin distribution could be altered by Fe, and which might account for the Fe-dependent root hair growth under -Pi.

Reduced sensitivity of -Pi-induced root hair growth to Fe availability, induced by the application of BFA

To validate the correlation between altered vesicle trafficking and root hair growth induced by Fe availability under $-\mathrm{Pi}$, we then observed the root hair growth treated with different Fe gradients under - $\mathrm{Pi}$ with the 
application of BFA. Different gradients of BFA and time-course root hair observation were performed. Results showed that the application of $500 \mathrm{nM}$ BFA notably inhibited root hair growth. And stronger repression was found as the application of $1500 \mathrm{nM} \mathrm{BFA} \mathrm{(Fig.} \mathrm{11;} \mathrm{Fig.} \mathrm{S5).} \mathrm{Under}+\mathrm{Pi}$, despite the application of BFA, the root hair growth remained sensitive to Fe (Fig. S5D). Under -Pi, when $500 \mathrm{nM}$ was applied, the sensitivity of root hair growth was significantly reduced at $3 \mathrm{~d}$ treatment, which was recovered as the extension of treatment. With the application of $1500 \mathrm{nM} \mathrm{BFA}$, the sensitivity of root hair growth was notably reduced throughout the observation (Fig. 11D). These results further indicated that the Fe-triggered root hair growth under $-\mathrm{Pi}$ is induced by the altered vesicle trafficking affected by Fe availability.

\section{Discussion}

Under sufficient and deficient $\mathrm{Pi}$, root hair growth is affected by the local Fe availability via separated processes

Understanding mechanisms of -Pi-induced root hair growth is urgent for improving the phosphate efficiency in agricultural systems. Recently, interactions between $\mathrm{Pi}$ and $\mathrm{Fe}$ on -Pi-induced root architecture remodeling have attracted increasing attention (Ward et al. 2008; Bouain et al. 2016; Gutiérrez-Alanís et al. 2017). To decipher the roles of $\mathrm{Fe}$ in root hair growth, especially under -Pi. We set different Fe gradients under $+\mathrm{Pi} /-\mathrm{Pi}$ for root hair observation. Here, we found the root hair growth was notably dependent on the Fe availability at both Pi levels (Fig. 1), and this process was induced by the local availability of Fe (Fig. 2). Previously, the increased root hair density has been noted as the most common morphological response to Fe deficiency (Schmidt et al. 2000; Schmidt \& Schikora 2001; Müller \& Schmidt 2004; Li \& Schmidt 2010). In this work, we suggested the dosage effects of $\mathrm{Fe}$ on root hair growth instead of being limited to the root hair density and Fe deficient condition. Meanwhile, with the morphological analysis of various mutants (Fig. 4; Fig. 5), which are discussed in the following parts, we found different processes are integrated into the Fe-triggered root hair growth under Pi sufficient and Pi deficient conditions. These results suggested the unique roles of $\mathrm{Fe}$ in -Pi-induced root hair growth, indicating the interplay between Fe and $\mathrm{Pi}$ in root hair growth.

ROS activity and cell wall-related process are probably involved in the Fe-mediated root hair growth under sufficient $\mathrm{Pi}$

Roles of each hormone in Fe-deficiency-induced morphological responses of root hairs have been discussed (Schmidt et al. 2000; Schmidt \& Schikora 2001; Müller \& Schmidt 2004). However, under +Pi, ein3eil1 , aux1-7, and arf10arf16 showed no distinct differences in sensitivity of root hair growth to Fe (Fig. 4; Table 1), implying that auxin and ethylene might not be required for the Fe-triggered root hair growth under $+\mathrm{Pi}$. In this work, ROS activity was positively correlated with the concentration of $\mathrm{Fe}$ (III) within the range of $0-350 \mu \mathrm{M}$ and remained unchanged as the concentration increasing under $+\mathrm{Pi}$ (Fig. 6), which is partially consistent with root hair growth. In proteomic analysis, proteins involved in the polysaccharide biosynthetic process were strongly affected by $\mathrm{Fe}$ under $+\mathrm{Pi}$. Abundances of proteins involved in the biosynthesis of cell wall components, including At3g62830 (UXS2) (Egelund et al. 2004), GAUT9 (Qinet al. 2013), SFR6 (Sorek et al. 2015), XEG113 (Gilleet al. 2009), UGE1 (Rösti et al. 2007), and MUR3 (Tedman-Jones et al. 2008) were significantly affected by Fe under +Pi (Fig. 7D). Among which, XEG113 (Gille et al. 2013; Velasquez et al. 2011), UGE1 (Seifert et al. 2004), and MUR3 (Xu et al. 2017) are essential for root hair growth or cell elongation. The decreased abundances of these proteins under $500 \mu \mathrm{M}$ Fe indicated that the high concentration of Fe (III) might inhibit the root hair growth via regulating the cell wall synthesis process. Above all, we suggested that under $+\mathrm{Pi}$, the root hair growth would be facilitated probably by enhancing the ROS activity and cell wall synthesis within 0-350 $\mu \mathrm{M}$ Fe, which would inhibit via repressing the synthesis cell wall as increasing of $\mathrm{Fe}(350-500 \mu \mathrm{M})$.

$\mathrm{Fe}$ availability triggers -Pi-induced root hair growth via altering vesicle trafficking

The proteomic analysis showed that proteins involved in vesicle trafficking were affected by $\mathrm{Fe}$, especially under -Pi (Fig. 7; Fig. 8). Proteins including GN (Fischer et al. 2006), ARF3 (Fischeret al. 2006) involved in budding; SEC12, SEC22, SEC3A, SEC23E, and SEC24 involved in tethering (Wen et al. 2005; Assaad 2008; Liet al. 2013), STX5, VAMP713, VAMP711, VTI1, and KEU (Assaadet al. 2001; Ichikawa et al. 
2014; Larson et al.2014) involved in membrane fusion were affected by Fe availability under -Pi (Fig. 7C; Fig. 8). The deduction supposed by proteomic analysis was confirmed by the application of BFA Under - $\mathrm{Pi}$, the vesicle trafficking process of seedlings treated with $20 \mu \mathrm{M}$ Fe displayed distinct resistance to the BFA treatment (Fig. 9). The reduced sensitivity of root hair growth to Fe availability under -Pi induced by BFA directly proved that the Fe affects -Pi-induced root hair growth by altering vesicle trafficking. Although the correlation between vesicle trafficking and root hair growth has been extensively discussed (Emons \& Ketelaar 2009; Žárský et al. 2009; Larson et al. 2014; Wangenheimet al. 2016), the involvement of vesicle trafficking in nutrient-induced root hairs growth is unclear. In our work, we found the Fe availability could affect vesicle trafficking under - $\mathrm{Pi}$ and subsequently regulating the root hair growth, indicating the potential role of vesicle trafficking in nutrient-induced root hair growth.

Fe availability modulates -Pi-induced root hair growth probably via altering auxin distribution

In this work, reduced sensitivity of root hair growth to Fe was observed in aux1-7 and arf10arf16, specifically under -Pi (Fig. 4), indicating auxin might be implicated in the Fe-mediated root hair growth under -Pi. In proteomic analysis, processes including auxin metabolism and auxin transport were enriched (Fig. 7) Proteins including GN, the GDP/GTP exchange factor for small G-proteins of the ADP ribosylation factor (RAF) class ( $\mathrm{Li}$ et al. , 2017) and is involved in the PIN1 asymmetric localization (Geldner et al. 2003); AXR4, which is required for the localization of AUX1 (Dharmasiri et al. 2006; Hobbie 2006); ASA1, a rate-limiting enzyme in tryptophan biosynthesis (Sun et al. 2009; Mao et al. 2016); AMI1, an enzyme involved in the biosynthesis of IAA (Nemoto et al. 2009; Sánchez-Parra et al. 2014; Pérez-Alonso et al. 2021), and ABCBs (ABCB5 and ABCB11), which are essential for auxin transport and iron homeostasis (Xu et al. 2014), were notably affected by the of Fe availability (Fig. 7E, F). These indicated the transport and metabolism of auxin might be affected by Fe under -Pi. The observation of fluorescence signals of DR5:GFP further confirmed the altered distribution of auxin signals (Fig. 10). These results suggested that auxin is required for the Fetriggered root hair growth under -Pi and probably due to the altered distribution affected by Fe availability. In addition, the polarized auxin transport is tightly correlated with the vesicle trafficking (Geldner et al. 2001; Whitewoods 2020), this prompt us to suppose that the Fe availability could affect vesicle trafficking, which in turn alters the auxin distribution, and subsequently regulating the root hair growth.

Fe availability alters phosphate starvation-induced responses and affects -Pi induced root hair growth via PHR1

In this work, reduced sensitivity of root hairs to Fe (III) was observed in phr1 under -Pi, indicating that PHR1 is required in the Fe-triggered root hair growth under -Pi (Fig. 5A, B; Table 1). With the detection of the relative expressions of PHR1 and its downstream PSR genes, we found the expressions of these genes were notably affected by Fe availability (Fig. 5E). PHR1 is not only the central regulator of phosphate starvation responses but also extensively integrated into the interplay between multiple nutrients (Briat et al. 2015).PHR1 could adjust the Fe homeostasis under -Pi via regulating FER1, which possessing PIBS cis-elements in its promoter (Briat et al. 2010; Bournier et al. 2013). Our results further revealed that besides induced by -Pi and subsequently modulating Fe homeostasis, the PHR1 could also respond to the Fe availability and trigger the root hair growth under -Pi (Rai et al. 2015; Fig. 5). The cross-talk between PHR1 and auxin, mediated by $A R F^{7}$ and $A R F 19$, has been uncovered (Huanget al. 2018). In our work, the auxin signaling was altered by Fe under -Pi. Thus, we hypothesized that the enhanced auxin signaling regulated by the availability of Fe (III) could promote the expression of PHR1, which subsequently works jointly with auxin on root hair growth. While whether the PHR1 is directly affected by Fe availability or by altered auxin signaling requires further investigation.

Model of the Fe-triggered root hair growth under Pi deprivation

The above results allowed us to draw a regulation model of the Fe-triggered root hair growth under -Pi (Fig. 12). The model listed in (Fig. 12) clearly illustrates how the root hair growth is enhanced at $20 \mu \mathrm{M}$ Fe under -Pi compared to the other two gradients of Fe. At $20 \mu \mathrm{M} \mathrm{Fe}$ under - $\mathrm{Pi}$, the process of vesicle trafficking would be enhanced and subsequently facilitate root hair growth (Assaad 2008). The transport of 
auxin is also enhanced probably caused by the altered vesicle trafficking (Whitewoods 2020). The enhanced expression of PHR1 and its downstream PSR genes might be affected by two possible ways, the enhanced auxin signaling pathway (Huang et al. 2018) or directly by the Fe availability. The auxin signaling works jointly withPHR1 (Bustos et al. 2010) on facilitating root hair growth by inducing the expression of RSL4 (Mangano et al. 2017) and its downstream root hair-related genes. And subsequently, the root hair growth would be notably enhanced at $20 \mu \mathrm{M}$ Fe under -Pi.

In this work, we found the dosage effects of Fe on root hair growth, and different processes are employed to regulate root hair growth at different $\mathrm{Pi}$ levels. We also uncovered the effects of Fe availability on vesicle trafficking, auxin distribution, and phosphate starvation responses under -Pi.We supposed that the availability of Fe could affect vesicle trafficking, which might subsequently alter the auxin distribution and PSR genes, and ultimately trigger the root hair growth under -Pi. Taken together, our work enriches the gaps of the role of iron in root hair growth under -Pi and enriches the understanding of the interplay between Fe and $\mathrm{Pi}$.

\section{Acknowledgments}

We sincerely appreciate Prof. Guang-Qin Guo (Lanzhou University, Lanzhou, China) and Dong-Wei Di (Institute of Soil Science, Chinese Academy of Sciences, Nanjing, China) for kindly offering seeds of aux1-7 and ein3eil1 ; Prof. Hong-Wei Guo (Southern University of Science and Technology, Guangdong, China) and Dolan L. (the University of Oxford, Oxford, United Kingdom) for kindly offering seeds of rsl4rsl2 and rhd6rsl1 ; and Prof. Zhao-Jun Ding (Shandong University, Shandong, China) for kindly providing seeds ofarf10arf16 and DRG:GFP. This research was funded by the National Natural Science Foundation of China (31370280, 31971625) and Research Fund of State Key Laboratory of Soil and Sustainable Agriculture, Institute of Soil Science, Chinese Academy of Sciences (Y812000006), and the Priority Academic Program Development of Jiangsu Higher Education Institutions (PAPD). Authors declare no competing interests.

\section{References}

Assaad F.F. (2008) The membrane dynamics of root hair morphogenesis. In: . Plant Cell Monographs . Berlin, Heidelberg: Springer.

Assaad F.F., Huet Y., Mayer U. \& Jürgens G. (2001). The cytokinesis gene KEULE encodes a Sec1 protein that binds the syntaxin knolle.J. Cell Biol. 152, 531-543.

Bates T.R. \& Lynch J.P. (2000). Plant growth and phosphorus accumulation of wild type and two root hair mutants of Arabidopsis thaliana (Brassicaceae). Am. J. Bot. 87, 958-63.

Bhosale R., Giri J., Pandey B.K., Giehl R.F., Hartmann A., Traini R., . . \& Swarup R. (2018). A mechanistic framework for auxin dependentArabidopsis root hair elongation to low external phosphate.Nat. Commun. 9, 1409 .

Bouain N., Doumas P. \& Rouached H. (2016). Recent advances in understanding the molecular mechanisms regulating the root system response to phosphate deficiency in Arabidopsis . Curr. Genomics 17, 308-314.

Bournier M., Tissot N., Mari S., Boucherez J., Lacombe E., Brait J. F. \& Gaymard F. ( 2013). Arabidopsis ferritin 1 (AtFer1) gene regulation by the phosphate starvation response 1 (AtPHR1) transcription factor reveals a direct molecular link between iron and phosphate homeostasis. J. Biol. Chem. 288, 22670-22680.

Briat J.F., Duc C., Ravet K. \& Gaymard F. (2010). Ferritins and iron storage in plants. Biochimi Biophys. 1800, 806-814.

Briat J.F., Rouached H., Tissot N., Gaymard F. \& Dubos C. (2015). Integration of P, S, Fe, and Zn nutrition signals in Arabidopsis thaliana : potential involvement of PHOSPHATE STARVATION RESPONSE 1 (PHR1). Front. Plant. Sci. 6, 290.

Bustos R., Castrillo G., Linhares F., Puga M.I., Rubio V., Perez-Perez J., . . \& Paz-Ares J. (2010). A central regulatory system largely controls transcriptional activation and repression responses to phosphate 
starvation in Arabidopsis . PLoS Genet.6, e1001102.

Carol R.J. \& Dolan L. (2006). The role of reactive oxygen species in cell growth: lessons from root hairs. J. Exp. Bot. 57,1829-1834.

Crombez H., Motte H. \& Beeckman T. (2019). Tackling plant phosphate starvation by the roots. Dev. Cell 48, 599-615.

Datta S., Prescott H. \& Dolan L. (2015). Intensity of a pulse of RSL4 transcription factor synthesis determines Arabidopsis root hair cell size. Nat. Plants 1, 15138.

Deng S., Sun J. Zhao R., Ding M., Zhang Y., Sun Y., .. \& \& Chen S. (2015). Populus euphratica APYRASE2 enhances cold tolerance by modulating vesicular trafficking and extracellular ATP inArabidopsis plants. Plant Physiol. 169, 530-548.

Dharmasiri S., Swarup R., Mockaitis K., Dharmasiri N., Singh S.K., Kowalchyk M., .. \& Estelle M. (2006). AXR4 is required for localization of the auxin influx facilitator AUX1. Science312, 1218-1220.

Dolgikh V.A., Pukhovaya E.M. \& Zemlyanskaya E.V. (2019). Shaping ethylene response: the role of EIN3/EIL1 transcription factors.Front. Plant. Sci. 10, 1030.

Dong J., Pineros M.A., Li X., Yang H., Liu Y., Murphy A.S., .. \& \& Liu D. (2017). An Arabidopsis ABC transporter mediates phosphate deficiency-induced remodeling of root architecture by modulating iron homeostasis in roots. Mol. Plant 10, 244-259.

Emons A.M.C. \& Ketelaar T. (2009). Intracellular Organization: A prerequisite for root hair elongation and cell wall deposition In: Emons AMC, Ketelaar T, eds Root Hairs , Berlin, Heidelberg: Springer, 27-44.

Egelund J., Skjot M., Geshi N. Ulvskov P. \& Petersen B.L. (2004). A complementary bioinformatics approach to identify potential plant cell wall glycosyltransferase-encoding genes. Plant Physiol.136, 2609-2620.

Estelle M.A., Somerville C. (1987). Auxin-resistant mutants of Arabidopsis thaliana with an altered morphology. Mol. Gen. Genet. 206, 200-206.

Feng Y., Xu P., Li B., Li P., Wen X., An F., .. \& \& Guo H. (2017). Ethylene promotes root hair growth through coordinated EIN3/EIL1 and RHD6/RSL1 activity in Arabidopsis . Proc. Natl. Acad. Sci. USA 114, 13834-13839.

Fischer U., Ikeda Y., Ljung K., Serralbo O., Singh M., Heidstra R., .. \& Grebe M. (2006). Vectorial information for Arabidopsis planar polarity is mediated by combined AUX1, EIN2, and GNOM activity. Curr Biol 16, 2143-2149.

Geldner N., Friml J., Stierhof Y.D., Jurgens G., Palme K. (2001). Auxin transport inhibitors block PIN1 cycling and vesicle trafficking. Nature 413, 425-428.

Geldner N., Anders N., Wolters H., Keicher J., Kornberger W., Muller P., .. \& Jurgens G. (2003). The Arabidopsis GNOM ARF-GEF mediates endosomal recycling, auxin transport, and auxin-dependent plant growth. Cell 112, 219-230.

Gille S., Hansel U., Ziemann M. \& Pauly M. (2009). Identification of plant cell wall mutants by means of a forward chemical genetic approach using hydrolases. Proc. Natl. Acad. Sci. USA 106,14699-14704.

Gille S., Sharma V., Baidoo E.E.K., Keasling J.D., Scheller H.V. \& Pauly M. (2013). Arabinosylation of a yariv-precipitable cell wall polymer impacts plant growth as exemplified by the Arabidopsisglycosyltransferase mutant ray1. Mol. Plant 6,1369-1372.

Gutierrez-Alanis D, Yong-Villalobos L, Jimenez-Sandoval P, Alatorre-Cobos F, Oropeza-Aburto A, MoraMacias J, ... \& Herrera-Estrella L. (2017). Phosphate starvation-dependent iron mobilization induces CLE14 expression to trigger root meristem differentiation through CLV2/PEPR2 signaling. Dev. Cell41, 555-570. 
Hobbie L.J. 2006. Auxin and cell polarity: the emergence of AXR4.Trends Plant Sci. 11, 517-518.

Huang J., Xue C., Wang H., Wang L., Schmidt W., Shen R., Lan P. (2017). Genes of ACYL CARRIER PROTEIN family show different expression profiles and overexpression of ACYL CARRIER PROTEIN 5 modulates fatty acid composition and enhances salt stress tolerance in Arabidopsis .Front. Plant Sci. 8, 987.

Huang K.L., Ma G.J., Zhang M.L., Xiong H., Wu H., Zhao C.Z., .. \& \& Ren F. (2018). The ARF7 and ARF19 transcription factors positively regulate PHOSPHATE STARVATION RESPONSE 1 in Arabidopsis roots. Plant Physiol. 178, 413-427.

Huang G. \& Zhang D. (2020). The plasticity of root systems in response to external phosphate. Int J Mol Sci 21, 5955 .

Ichikawa M., Hirano T., Enami K., Fuselier T., Kato N., Kwon C., . . \& Sato M.H. (2014). Syntaxin of plant proteins SYP123 and SYP132 mediate root hair tip growth in Arabidopsis thaliana. Plant Cell Physiol.55, 790-800.

Lan P., Li W. \& Schmidt W. (2012). Complementary proteome and transcriptome profiling in phosphatedeficient Arabidopsis roots reveals multiple levels of gene regulation. Mol. Cell Proteomics11, 1156-1166.

Larson E.R., Domozych D.S. \& Tierney M.L. (2014). SNARE VTI13 plays a unique role in endosomal trafficking pathways associated with the vacuole and is essential for cell wall organization and root hair growth in Arabidopsis . Ann. Bot. 114, 1147-1159.

Li R., Rodriguez-Furlan C., Wang J., van de Ven W., Gao T., Raikhel NV. \& Hicks GR. (2017). Different endomembrane trafficking pathways establish apical and basal polarities. Plant Cell 29,90-108.

Li S., Chen M., Yu D., Ren S., Sun S., Liu L., .. \& \& Liu C.M. (2013). EXO70A1-mediated vesicle trafficking is critical for tracheary element development in Arabidopsis . Plant Cell 25:1774-1786.

Li W. \& Schmidt W. (2010). A lysine-63-linked ubiquitin chain-forming conjugase, UBC13, promotes the developmental responses to iron deficiency in Arabidopsis roots. Plant J. 62,330-343.

Ma Z., Bielenberg D.G., Brown K.M. \& Lynch J.P. (2001). Regulation of root hair density by phosphorus availability in Arabidopsis thaliana. Plant, Cell and Environment 24, 459-467.

Maher E.P. \& Martindale S.J. (1980). Mutants of Arabidopsis thaliana with altered responses to auxins and gravity. Biochem Genet. 18, 1041-1053.

Mangano S., Denita-Juarez S.P., Choi H.S., Marzol E., Hwang Y., Ranocha R., .. \& Estevez J.M. (2017). Molecular link between auxin and ROS-mediated polar growth. Proc. Natl. Acad. Sci. USA114, 5289-5294.

Mao J.L., Miao Z.Q., Wang Z., Yu L.H., Cai X.T. \& Xiang C.B. (2016).Arabidopsis ERF1 mediates crosstalk between ethylene and auxin biosynthesis during primary root elongation by regulating ASA1 expression. PLoS Genet. 12, e1006076.

Mendrinna A. \& Persson S. (2015). Root hair growth: it's a one way street. F1000Prime Rep. 7, 23.

Mittler R. (2006). Abiotic stress, the field environment and stress combination. Trends Plant Sci. 11, 15-19.

Muller J., Toev T., Heisters M., Teller J., Moore K.L., Hause G., .. \& \& Abel S. (2015). Iron-dependent callose deposition adjusts root meristem maintenance to phosphate availability. Dev. Cell 33,216-230.

Muller M. \& Schmidt W. (2004). Environmentally induced plasticity of root hair development in Arabidopsis . Plant Physiol.134, 409-419.

Nemoto K., Hara M., Suzuki M., Seki H., Muranaka T. \& Mano Y. (2009). The NtAMI1 gene functions in cell division of tobacco BY-2 cells in the presence of indole-3-acetamide. FEBS Lett. 583, 487-492. 
Niu Y.F., Chai R.S., Jin G.L., Wang H., Tang C.X. \& Zhang Y.S. 2013. Responses of root architecture development to low phosphorus availability: a review. Ann. Bot. 112, 391-408.

Ovečka M., Lang I., Baluška F., Ismail A., Illeš P. \& Lichtscheidl IK. (2005). Endocytosis and vesicle trafficking during tip growth of root hairs. Protoplasma 226, 39-54.

Parry G. (2018). Low phosphate puts auxin in the root hair. Trends Plant Sci. 23, 845-847.

Pérez-Alonso M.M., Ortiz-García P., Moya-Cuevas J., Lehmann T., Sánchez-Parra B., Björket R.G., ... \& Pollmann S. (2021). Endogenous indole-3-acetamide levels contribute to the crosstalk between auxin and abscisic acid, and trigger plant stress responses in Arabidopsis .J. Exp. Bot. 72, 459-475.

Qin L.X., Rao Y., Li L., Huang J..F, Xu W.L. \& Li X.B. (2013). Cotton GalT1 encoding a putative glycosyltransferase is involved in regulation of cell wall pectin biosynthesis during plant development. PLoS One 8, e59115.

Rahman A., Hosokawa S., Oono Y., Amakawa T., Goto N. \& Tsurumi S. (2002). Auxin and ethylene response interactions during Arabidopsis root hair development dissected by auxin influx modulators. Plant Physiol. 130, 1908-1917.

Reyt G., Boudouf S., Boucherez J., Gaymard F. \& Briat J.F. (2014). Iron and ferritin dependent ROS distribution impact Arabidopsis root system architecture. Mol. Plant 9, 133.

Rösti J., Barton C.J., Albrecht S., Dupree P., Pauly M., Findlay K., . . \& Seifert G.J. (2007). UDP-glucose 4-epimerase isoforms UGE2 and UGE4 cooperate in providing UDP-galactose for cell wall biosynthesis and growth of Arabidopsis thaliana. Plant Cell 19,1565-1579.

Rouached H., Secco D. \& Arpat B.A. (2010). Regulation of ion homeostasis in plants: Current approaches and future challenges.Plant signaling $\mathscr{E}$ behavior 5, 501-502.

Sánchez-Parra B., Frerigmann H., Alonso M.M.P., Loba V.C., Jost R., Hentrich M. \& Pollmann S. (2014). Characterization of four bifunctional plant IAM/PAM-amidohydrolases capable of contributing to auxin biosynthesis. Plants (Basel) 3, 324-347.

Saiz-Fernández I., Černý M., Skalák J. \& Brzobohatý B. (2021). Split-root systems: detailed methodology, alternative applications, and implications at leaf proteome level. Plant Methods 17,7.

Schmidt W. \& Schikora A. (2001). Different pathways are involved in phosphate and iron stress-induced alterations of root epidermal cell development. Plant Physiol. 125, 2078-2084.

Schmidt W., Tittel J. \& Schikora A. (2000). Role of hormones in the induction of iron deficiency responses in Arabidopsis roots. Plant Physiol. 122, 1109-1118.

Seifert G.J., Barber C., Wells B. \& Roberts K. (2004). Growth regulators and the control of nucleotide sugar flux. Plant Cell24, 723-730.

Shibata M. \& Sugimoto K. (2019). A gene regulatory network for root hair development. J. Plant Res. 132, 301-309.

Sogawa A., Yamazaki A., Yamasaki H., Komi M., Manabe T., Tajima S., .. \& Nomura M. (2019). SNARE proteins LjVAMP72a and LjVAMP72b are required for root symbiosis and root hair formation in Lotus japonicus .Front. Plant Sci. 16, 1992.

Sorek N., Szemenyei H., Sorek H., Landers A., Knight H., Bauer S., . . \& Somerville C.R. (2015). Identification of MEDIATOR16 as theArabidopsis COBRA suppressor MONGOOSE1. Proc. Natl. Acad. Sci. USA 112, 16048-16053.

Sun J., Xu Y.,Ye S., Jiang H., Chen Q., Liu F., .. \& \& Li C. (2009).Arabidopsis ASA1 is important for jasmonate-mediated regulation of auxin biosynthesis and transport during lateral root formation. Plant Cell 21, 1495-1511. 
Tedman-Jones J.D., Lei R., Jay F., Fabro G., Li X., Reiter W.D., . . \& Jones J.D.G. (2008). Characterization of Arabidopsis mur3 mutations that result in constitutive activation of defence in petioles, but not leaves. Plant J. 56, 691-703.

Velasquez S.M., Barbez E., Kleine-Vehn J. \& Estevez J.M. (2016). Auxin and cellular elongation. Plant physiol. 170, 1206-1215.

Velasquez S.M., Ricardi M.M. \& Dorosz J.G. (2011). O-Glycosylated cell wall proteins are essential in root hair growth. Science332, 1401-1403.

Vijayakumar P., Datta S. \& Dolan L. (2016). ROOT HAIR DEFECTIVE SIX-LIKE4 (RSL4) promotes root hair elongation by transcriptionally regulating the expression of genes required for cell growth. New Phytol. 212, 944-953.

Vissenberg K., Claeijs N., Balcerowicz D. \& Schoenaers S. (2020). Hormonal regulation of root hair growth and responses to the environment in Arabidopsis thaliana . J. Exp. Bot. 71,2412-2427.

Wang J.W., Wang L.J., Mao Y.B., Cai W.J., Xue H.W. \& Chen X.Y. (2005). Control of root cap formation by MicroRNA-targeted auxin response factors in Arabidopsis . Plant Cell 17, 2204-2216.

Wangenheim D.V., Rosero A., Komis G., Šamajová O., Ovečka M., Voigt B. \& Šamaj J. (2016). Endosomal interactions during root hair growth. Front. Plant Sci. 6, 1262.

Ward J.T., Lahner B., Yakubova E., Salt D.E. \& Raghothama K.G. (2008). The effect of iron on the primary root elongation of Arabidopsisduring phosphate deficiency. Plant Physiol. 147,1181-1191.

Wen T.J., Hochholdinger F., Sauer M., Bruce W. \& Schnable P.S. (2005). The roothairless1 gene of maize encodes a homolog of sec3, which is involved in polar exocytosis. Plant Physiol. 138,1637-1643.

Whitewoods C. (2020). Flipping the Vs: Integrating vesicle trafficking, pin polarity, and plant development. Plant Cell 32,1354.

Xu J. \& Scheres B. (2005). Dissection of ArabidopsisADP-RIBOSYLATION FACTOR 1 function in epidermal cell polarity. Plant cell $\mathbf{1 7}, 525-536$.

Xu Y., Zhang S., Guo H., Wang S., Xu L., Li C., .. \& \& Jiang D.A. (2014). OsABCB14 functions in auxin transport and iron homeostasis in rice (Oryza sativa L ). Plant J. 79, 106-117.

Xu Z., Wang M., Shi D., Zhou G., Niu T., Hahn MG., .. \& \& Kong Y. (2017). DGE-seq analysis of MUR3related Arabidopsis mutants provides insight into how dysfunctional xyloglucan affects cell elongation. Plant Sci. 258, 156-169.

Yan M., Xue C., Xiong Y., Meng X., Li B., Shen R. \& Lan P. (2020). Proteomic dissection of the similar and different responses of wheat to drought, salinity and submergence during seed germination. J. Proteomics 220, 103756.

Yi K., Menand B., Bell E. \& Dolan L. (2010). A basic helix-loop-helix transcription factor controls cell growth and size in root hairs.Nat. Genet. 42, 264-267.

Žárský V., Cvrčková F., Potocký M. \& Hála M. (2009). Exocytosis and cell polarity in plants-exocyst and recycling domains. New Phytol. 183, 255-272.

Zeb Q., Wang X. \& Hou C. (2020). The interaction of CaM7 and CNGC14 regulates root hair growth in Arabidopsis. J. Integr. Plant Biol. 62, 887-896.

Zhou X., Xiang Y., Li C. \& Yu G. (2020). Modulatory role of reactive oxygen species in root development in model plant of Arabidopsis thaliana . Front. Plant Sci. 11, 485932.

Zhou Y., Zhou B., Pache L., Chang M., Khodabakhshi A.H., Tanaseichuk O., .. \& \& Chanda S.K. (2019). Metascape provides a biologist-oriented resource for the analysis of systems-level datasets. Nat. Commun.10, 
1523.

Tables

Table 1 Effects of Fe availability on root hair length of wild type and mutants.

\begin{tabular}{llllll}
\hline Material & Pi Sufficient (+Pi) & Pi Sufficient (+Pi) & Pi Sufficient (+Pi) & Pi Sufficient (+Pi) & Pi Sufficien \\
& $\mathbf{0} \boldsymbol{\mu M} \mathbf{\Phi} \boldsymbol{\varepsilon}$ & $\mathbf{3 5 0} \boldsymbol{\mu M} \mathbf{\Phi} \boldsymbol{\varepsilon}$ & $\mathbf{5 0 0} \boldsymbol{\mu M} \mathbf{\Phi} \boldsymbol{\varepsilon}$ & {$[?](350-0)$} & {$[?](350-500)$} \\
WT & $0074 \pm 002$ & $0155 \pm 003$ & $009 \pm 002$ & $0079 \pm 003$ & $0064 \pm 001$ \\
ein3eil1 & $0066 \pm 003$ & $0123 \pm 004$ & $0054 \pm 002$ & $0065 \pm 002$ & $0073 \pm 004$ \\
aux1-7 & $0055 \pm 003$ & $0127 \pm 003$ & $0081 \pm 003$ & $0066 \pm 003$ & $0048 \pm 0002$ \\
arf10 arf16 & $0073 \pm 004$ & $0161 \pm 002$ & $0096 \pm 003$ & $0086 \pm 003$ & $0064 \pm 002$ \\
phr1 & $0086 \pm 002$ & $0155 \pm 002$ & $0086 \pm 002$ & $008 \pm 003$ & $0068 \pm 002$ \\
rsl4 rsl2 & - & - & - & - & - \\
rhd6 rsl1 & - & - & - & - & - \\
\hline
\end{tabular}

Values represent root hair length $(\mathrm{mm})(\operatorname{mean} \pm \mathrm{SD})$ were data from one biological repeat, and totally at least three biological repeated were performed. Values of 'average [?]' ( $\mathrm{mm}$ ) (mean+-SD), the relative root hair length, were data averaged by all biological repeats. Significant differences between mutants and the WT under the same treatment were analyzed by t-test $\left(*, \mathrm{P}\right.$-Value $<0.05,{ }^{* *}, \mathrm{P}$-Value $\left.<0.01\right)$. -: represents no root hairs were observed.

\section{Figure Legends}

Fig. 1 Fe-triggered root hair growth under Pi sufficient and Pi deficient conditions. (A) and (B) Representative images of root hairs treated with different Fe gradients under Pi sufficient and Pi deficient conditions. Scare bar $=500 \mu \mathrm{m}$. (C) and (D) Estimation of root hair length and root hair density of each treatment. Data are means \pm SD of three biological repeats. Statistical analysis was performed by two-way ANOVA, and different letters indicate significant differences with P-Value $<0.05$.

Fig. 2 Local Fe availability triggers root hair growth. (A) Images of the split-root experiment of each pair of Fe gradients. Scale bar=1 cm. (B) Images of the root hairs of each pair of Fe gradients in the split-root experiment. Scare bar $=500 \mu \mathrm{m}$. Estimation of root hair length (left) and root hair density (right) of each pair of Fe gradients. (C) +Pi+0 Vs. $+\mathrm{Pi}+350(\mu \mathrm{M} \mathrm{Fe}) ;(\mathrm{D})+\mathrm{Pi}+0 \mathrm{Vs} .+\mathrm{Pi}+500(\mu \mathrm{M} \mathrm{Fe}) ;(\mathrm{E})+\mathrm{Pi}+350$ Vs. $+\mathrm{Pi}+500(\mu \mathrm{M} \mathrm{Fe}) ;(\mathrm{F})-\mathrm{Pi}+0$ Vs. $-\mathrm{Pi}+20(\mu \mathrm{M} \mathrm{Fe}) ;(\mathrm{G})-\mathrm{Pi}+0$ Vs. $-\mathrm{Pi}+100(\mu \mathrm{M} \mathrm{Fe}) ;(\mathrm{H})-\mathrm{Pi}+20 \mathrm{Vs}$. $-\mathrm{Pi}+100(\mu \mathrm{M} \mathrm{Fe})$; Data are means $\pm \mathrm{SD}$. Three biological repeats are performed and data of one is displayed. Statistical analysis was performed by Student's test, ${ }^{(*}$ ' indicated significant differences with $\mathrm{P}$-value $<0.05$, '**' indicated significant differences with $\mathrm{P}$-value $<0.01$.

Fig. 3 Effects of Fe availability on the root hair growth ofrsl4rsl2 and rhd6rsl1. Representative images of root hairs of (A) WT, (B) rsl4rsl2 , and (C) rhd6rsl1 treated with different Fe gradients under Pi sufficient and Pi deficient conditions. Scare bar $=500 \mu \mathrm{m}$. (D) Heatmap representing the relative expression of RSL4 and its downstream genes. Three biological repeats are performed and data of one is displayed.

Fig. 4 Effects of Fe availability on the root hair growth of hormone-related mutants. Representative images of root hairs of (A) WT, (C) aux1-7, (E) arf10arf16, and (G) ein3eil1, treated with different Fe gradients under Pi sufficient and Pi deficient conditions. Scare bar=500 $\mu \mathrm{m}$. Root hair length of each mutant (B) WT, (D) $\operatorname{aux1-7},(\mathrm{F}) \operatorname{arf10arf16},(\mathrm{H})$ ein3eil1 . Data are means \pm SD. For each mutant at least three biological repeats are performed and data of one is displayed. Statistical analysis was performed by two-way ANOVA, and different letters indicate significant differences with $\mathrm{P}$-Value $<0.05$.

Fig. 5 Effects of Fe availability on the root hair growth of $p h r 1$. Representative images of root hairs of (A) WT and (B) phr1, treated with different Fe gradients under Pi sufficient and Pi deficient conditions. Scare bar $=500 \mu \mathrm{m}$. Estimation of root hair length of each mutant, (C) WT and (D) phr1 . Data are means \pm SD. For each mutant at least three biological repeats are performed and data of one is displayed. Statistical 
analysis was performed by two-way ANOVA, and different letters indicate significant differences with PValue $<0.05$. (E) Heatmap representing the relative expression of PHR1 and its downstream genes. Three biological repeats are performed and data of one is displayed.

Fig. 6 Effects of Fe availability on the ROS activity. ROS activity of roots treated with Fe gradients under $\mathrm{Pi}$ sufficient and $\mathrm{Pi}$ deficient conditions stained by H2DCF-DA, (A images in white light; (B) images of fluorescence in dark. Estimation of the integrate optical density representing ROS activity of each treatment, under (C) sufficient Pi and (D) deficient Pi. Scare bar=100 $\mu \mathrm{m}$. Data are means \pm SD of three biological repeats. Statistical analysis was performed by one-way ANOVA, and different letters indicate significant differences with $\mathrm{P}$-Value $<0.05$.

Fig. 7 GO enrichment analysis of DAPs between different Fe gradients at each Pi level. (A) Bubble chart of representative GO terms enriched by DAPs of 350Vs.0 ( $\mu \mathrm{M} \mathrm{Fe})$ and $350 \mathrm{Vs} .500(\mu \mathrm{M} \mathrm{Fe})$ under +Pi. GO terms enriched by DAPs of 350Vs.0 ( $\mu \mathrm{M} \mathrm{Fe}$ ) were red circles and GO terms enriched by DAPs of 350Vs.500 $(\mu \mathrm{M} \mathrm{Fe})$ were blue squares. (B) Bubble chart of representative GO terms enriched by DAPs of $20 \mathrm{Vs} .0(\mu \mathrm{M}$ $\mathrm{Fe}$ ) and 20Vs.100 ( $\mu \mathrm{M} \mathrm{Fe}$ ) under -Pi. GO terms enriched by DAPs of 20Vs.0 ( $\mu \mathrm{M} \mathrm{Fe}$ ) were red circles and GO terms enriched by DAPs of 20Vs.100 ( $\mu \mathrm{M} \mathrm{Fe}$ ) were blue squares. Size of the bubbles represented the numbers enriched in each GO terms. Heatmaps of proteins enriched in processes including $(\mathrm{C})$ Golgi vesicle transport, (D) polysaccharide biosynthetic process, (E) auxin transport, (F) auxin metabolism, and (G) root epidermal cell differentiation.

Fig. 8 The sketch map of the SNARE interaction in vesicular transport marked with enriched DAPs. Abundances of proteins enriched in the KEGG pathway: SNARE interaction in vesicular transport were displayed. Yellow boxes represented enriched DAPs. Heatmaps represented the abundances of each protein under each treatment and DAPs enriched at different Pi levels were represented with different colors.

Fig. 9 The observation of vesicle trafficking in seedlings treated with different Fe gradients under Pi sufficient and Pi deficient conditions. (A) Images of cells stained by FM-4-64 for $10 \mathrm{~min}$. Scare bar=20 $\mu \mathrm{m}$. Effects of BFA on vesicle trafficking of seedlings treated with different Fe gradients, after treating with BFA for 1.5 $\mathrm{h}$, under $(\mathrm{B})+\mathrm{Pi}$ and $(\mathrm{F})$-Pi. Effects of BFA on vesicle trafficking of seedlings treated with different $\mathrm{Fe}$ gradients, after removing BFA for $1.5 \mathrm{~h}$, under $(\mathrm{C})+\mathrm{Pi}$ and $(\mathrm{G})-\mathrm{Pi}$. Red spots were BFA bodies. Scare $\mathrm{bar}=20 \mu \mathrm{m}$. Estimation of the average sizes of the BFA bodies of seedlings treated with different Fe gradients, after treating with BFA for $1.5 \mathrm{~h}$, under $(\mathrm{D})+\mathrm{Pi}$ and $(\mathrm{H})-\mathrm{Pi}$. Estimation of the average sizes of the BFA bodies of seedlings treated with different Fe gradients, after removing BFA for $1.5 \mathrm{~h}$, under (E) +Pi and (I) -Pi. Data are means $\pm \mathrm{SD}$, at least three biological repeats have been performed and data of one is displayed. Statistical analysis was performed by one-way ANOVA. Different letters indicate significant differences with P-Value $<0.05$.

Fig. 10 The observation of auxin distribution in seedlings treated with different Fe gradients. Effects of Fe availability on auxin distribution under Pi sufficient and $\mathrm{P}$ deficient conditions. (A) Images of fluorescence of DR5:GFP. From top to button are images under white light, images under dark light, and merged images. Scare bar $=20 \mu \mathrm{m}$. Estimation of the fluorescence signal ranges of each treatment under (B) $+\mathrm{Pi}$ and $(\mathrm{C})-\mathrm{Pi}$. Data are means $\pm \mathrm{SD}$, two biological repeats have been performed and data of one is displayed. Statistical analysis was performed by Student's test. '*' indicated significant differences with $\mathrm{P}$-value $<0.05$, '**, indicated significant differences with $\mathrm{P}$-value $<0.01$.

Fig. 11 The effects of BFA on Fe-triggered root hair growth under Pi deficient condition. Time-course observation of root hairs grown under different Fe gradients treated with (A) $0 \mathrm{nM}$ BFA, (B) $500 \mathrm{nM}$ BFA, and (C) $1500 \mathrm{nM}$ BFA. Scale bar $=500 \mu \mathrm{m}$. (D) Root hair length of each treatment at each time point. Data are the means \pm SD of two biological repeats. Statistical analysis was performed by Student's test. '*' indicated significant differences with $\mathrm{P}$-value $<0.05,{ }^{\prime} * *$ ' indicated significant differences with $\mathrm{P}$-value $<$ 0.01 .

Fig. 12 The model of the regulatory network that explains the Fe-triggered root hair growth under Pi deprivation. Under Pi deprivation, the vesicle trafficking, auxin distribution, and the expression of PHR1 
would be affected by the Fe availability. The vesicle trafficking would be notably enhanced at $20 \mu \mathrm{M} \mathrm{Fe}$ and subsequently facilitate root hair growth. The enhanced vesicle trafficking might facilitate the auxin transport, which induces the expression of PHR1 afterward. The enhanced auxin signaling, as well as the PHR1, would work jointly on facilitating root hair growth under -Pi. For more details, see "Discussion".

A

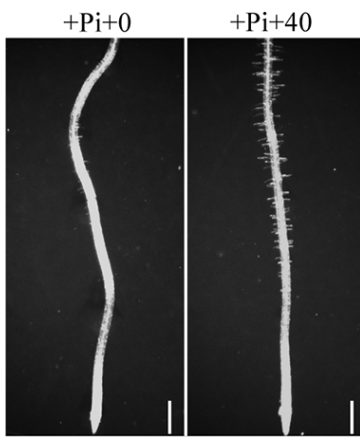

$+\mathrm{Pi}+150$

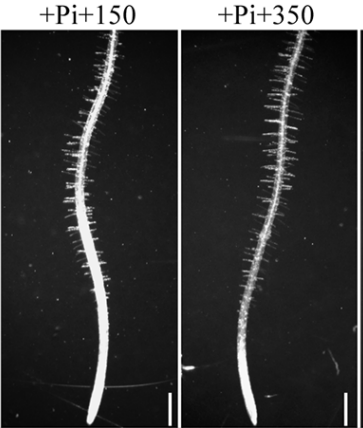

$+\mathrm{Pi}+500 \quad(\mu \mathrm{MFe})$

B
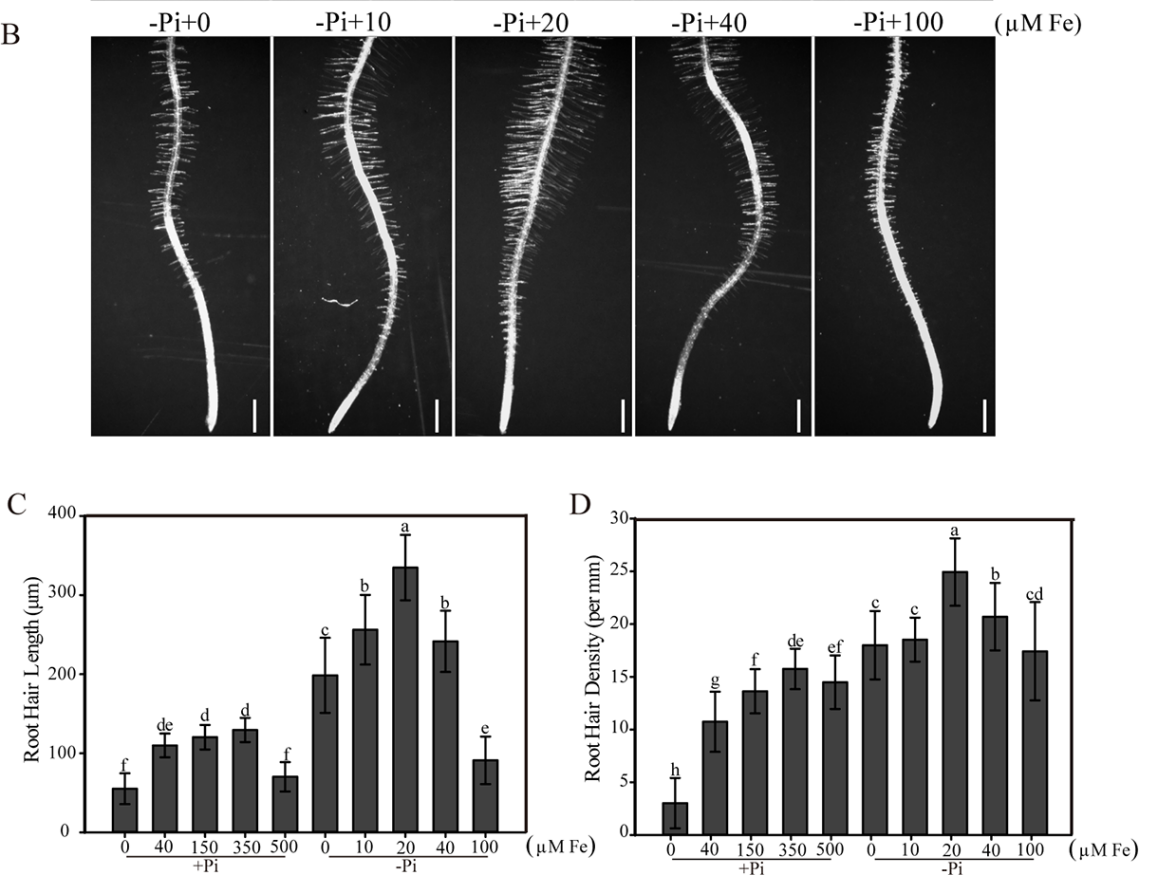

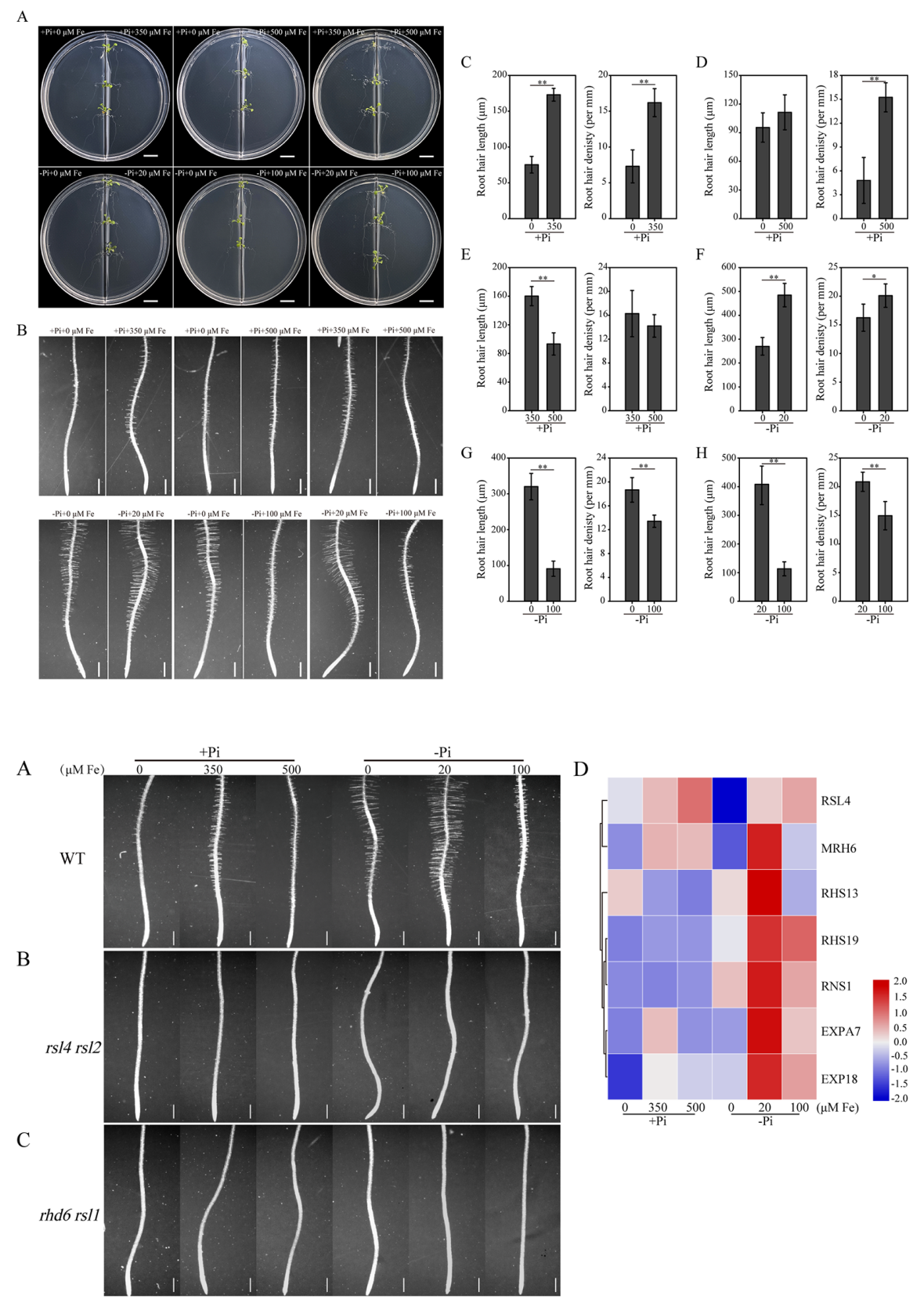

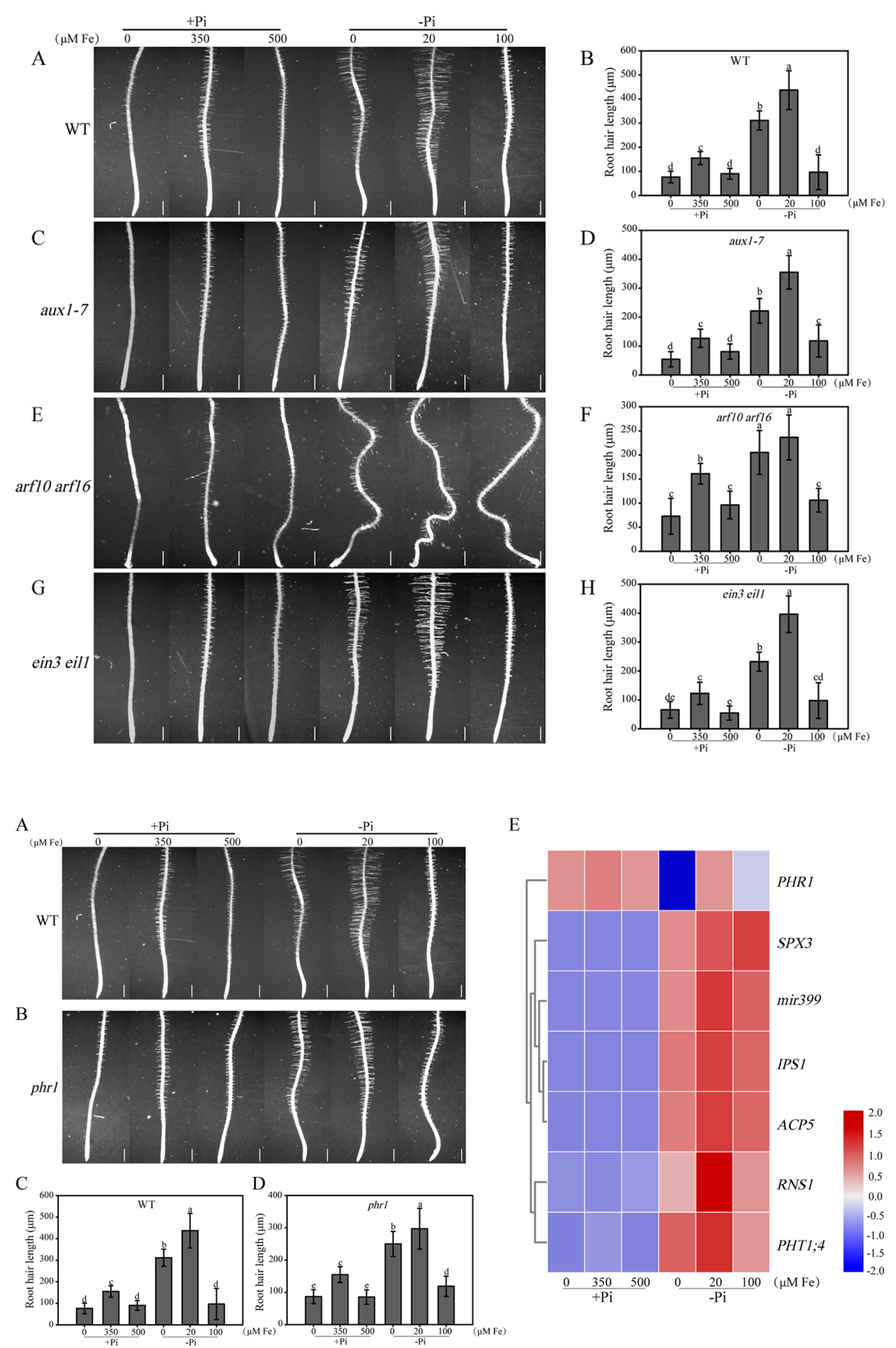

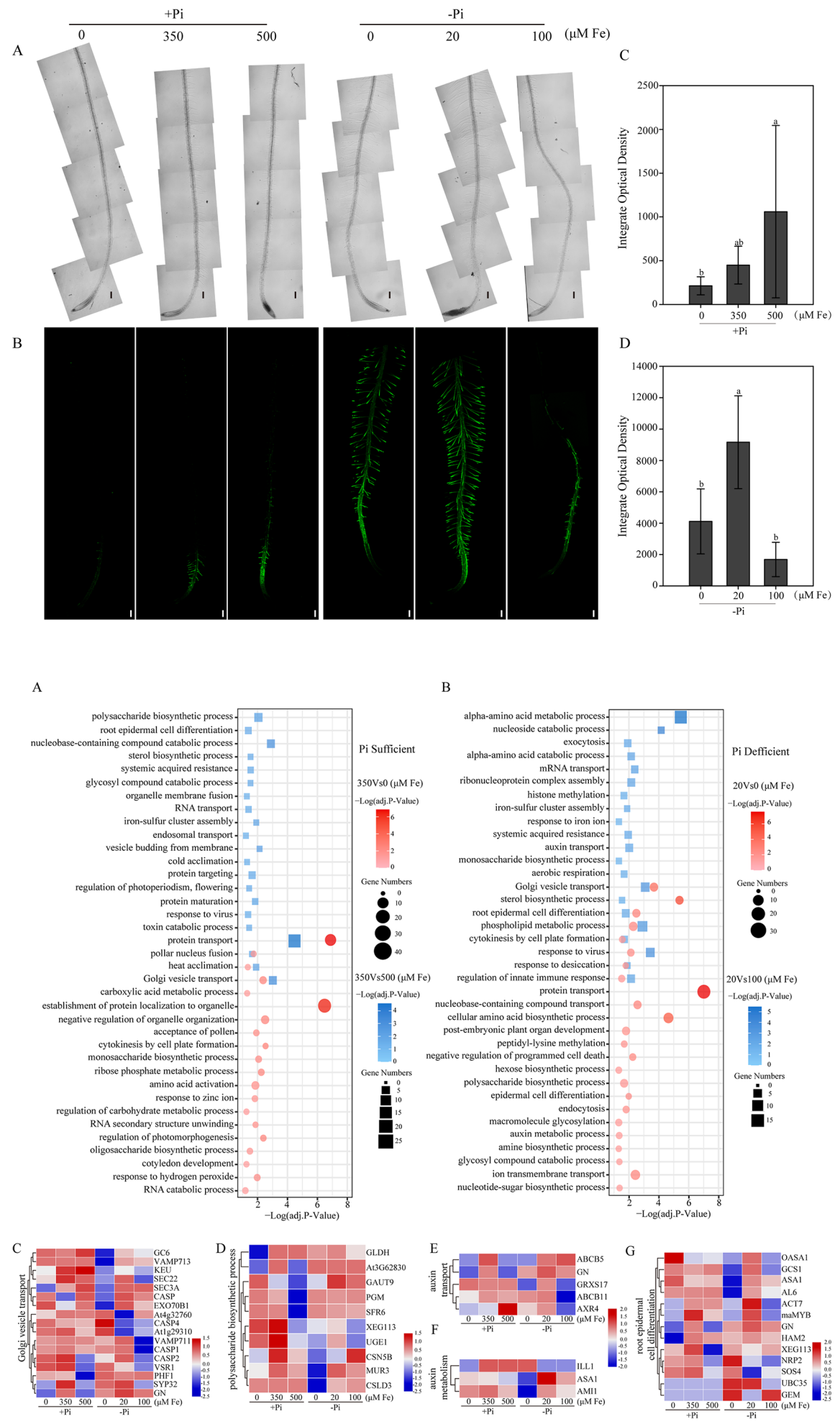


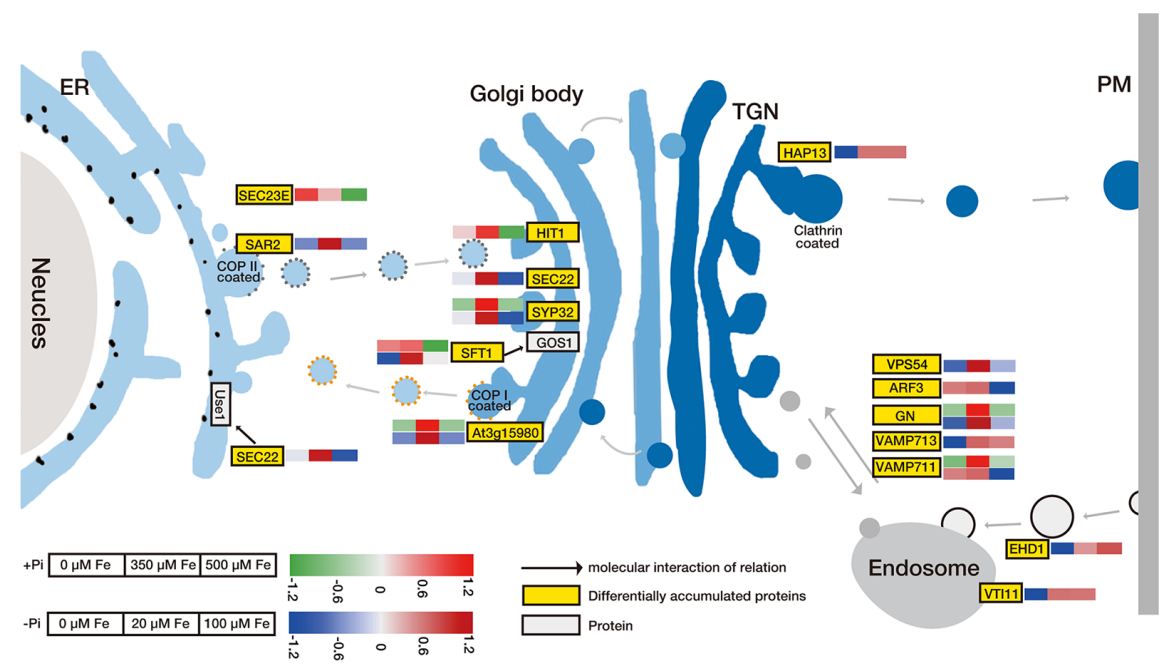




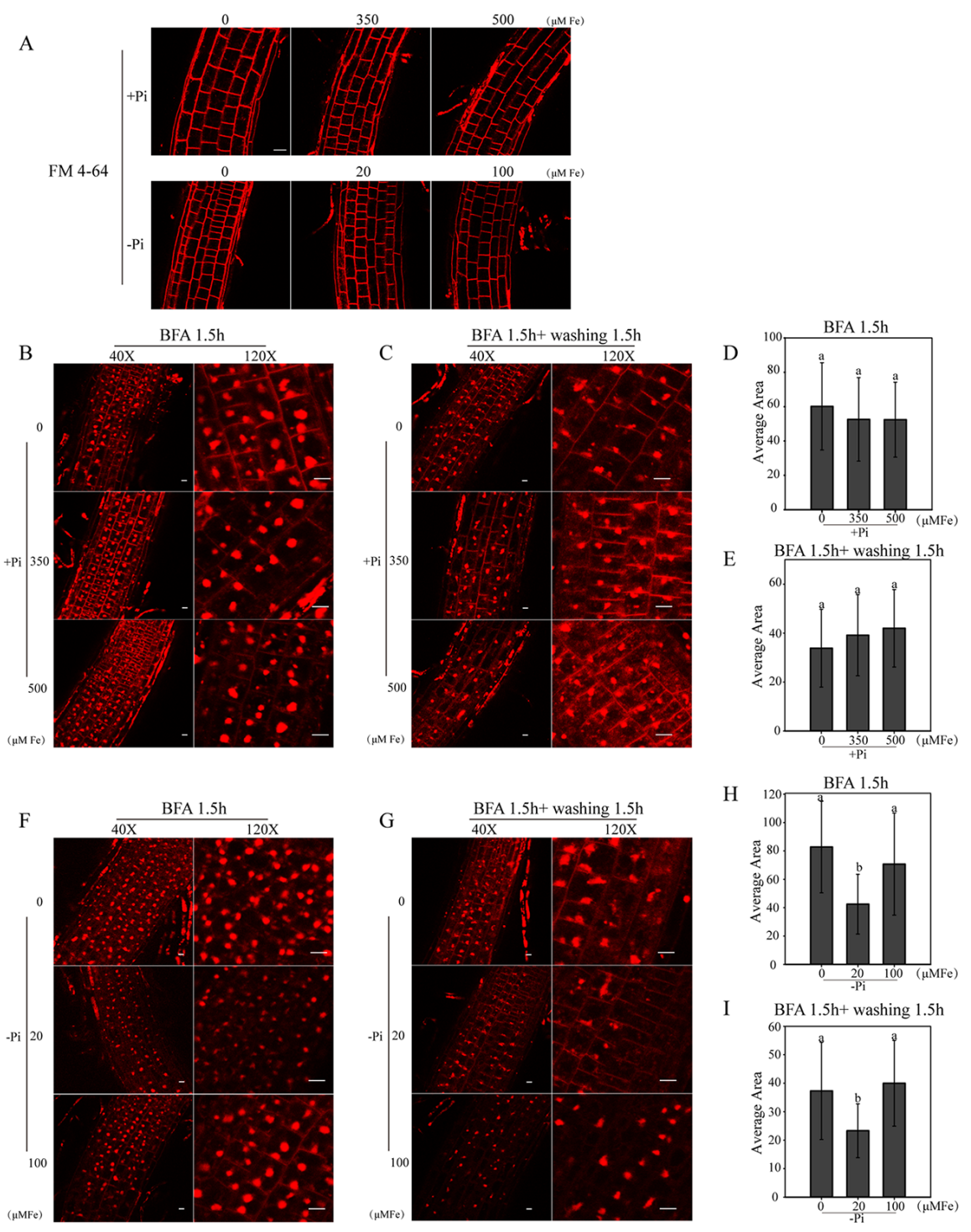



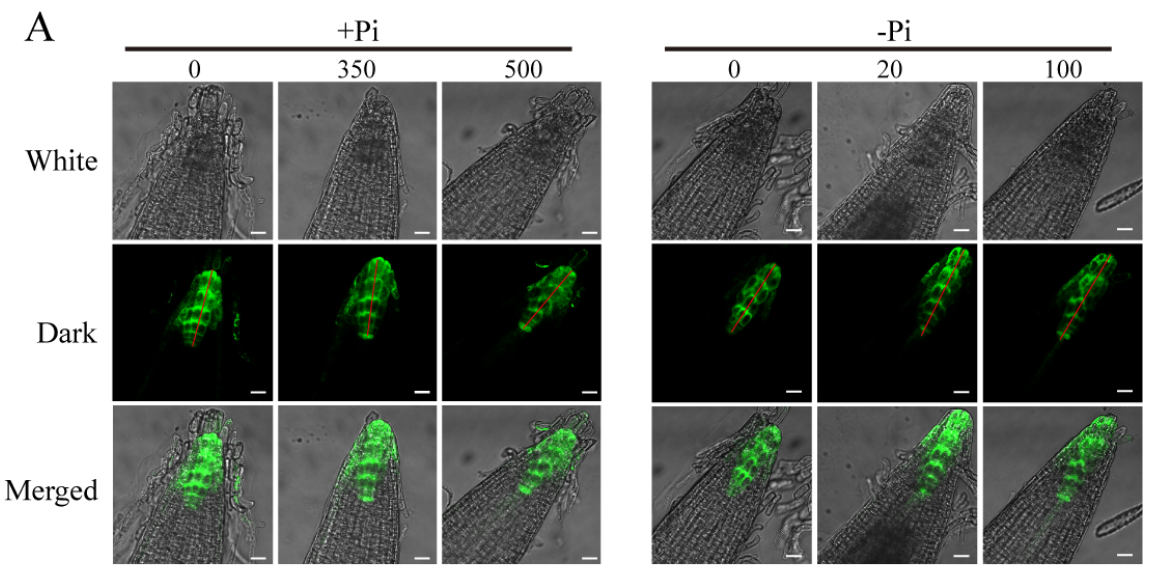

B
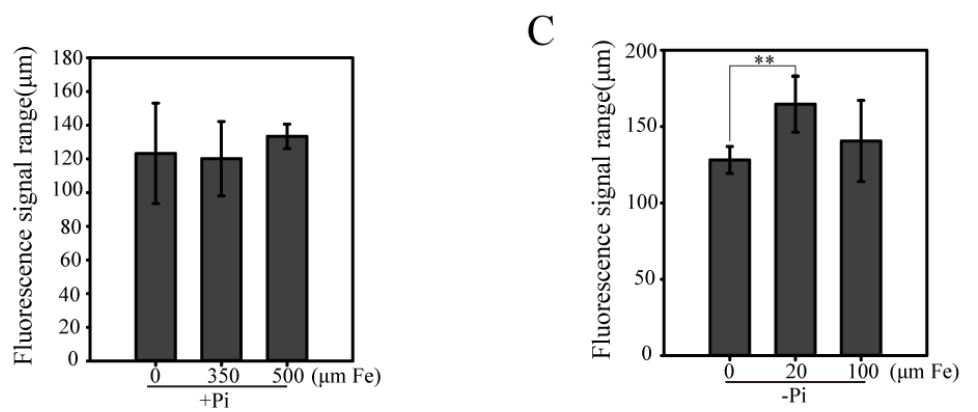


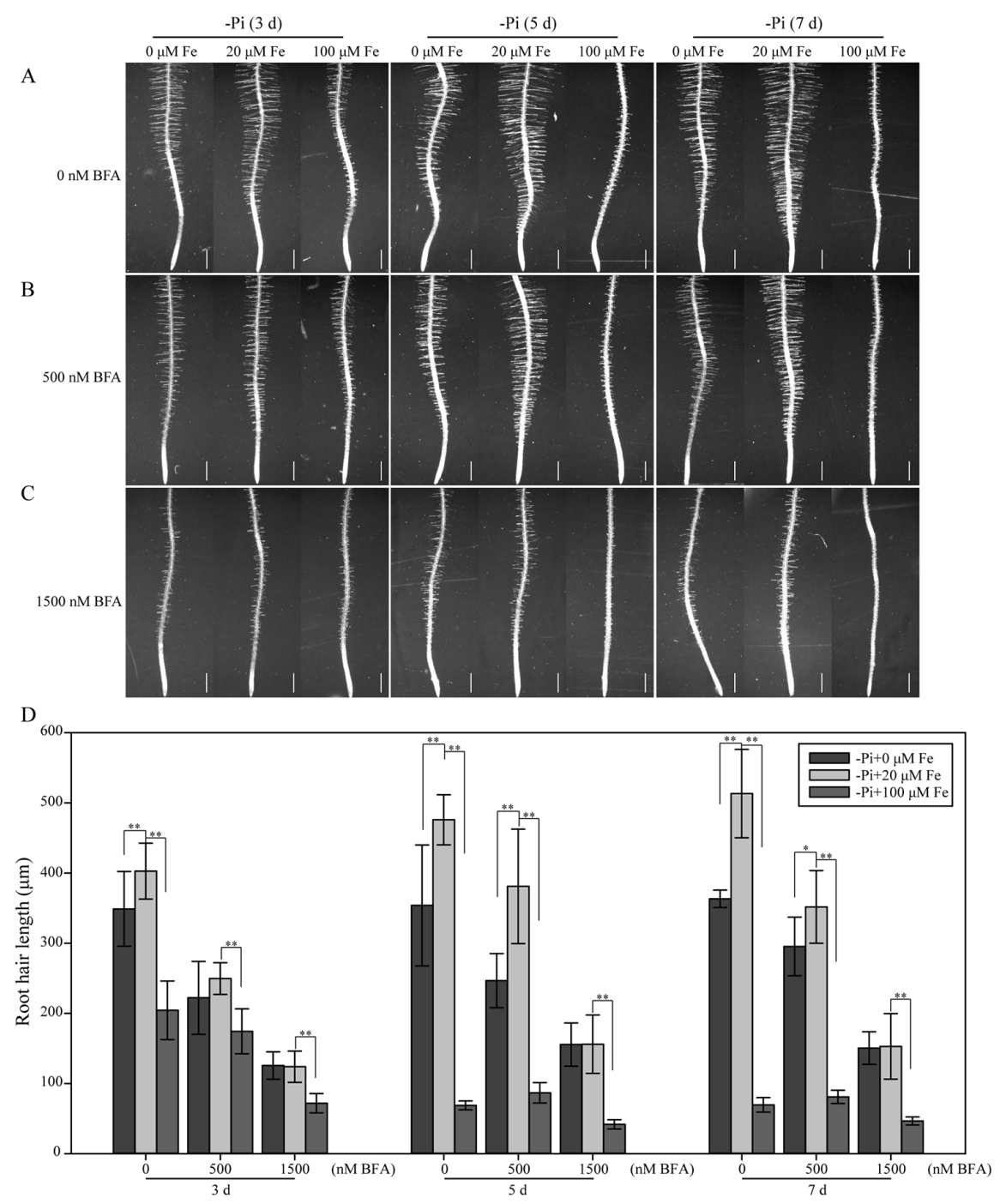




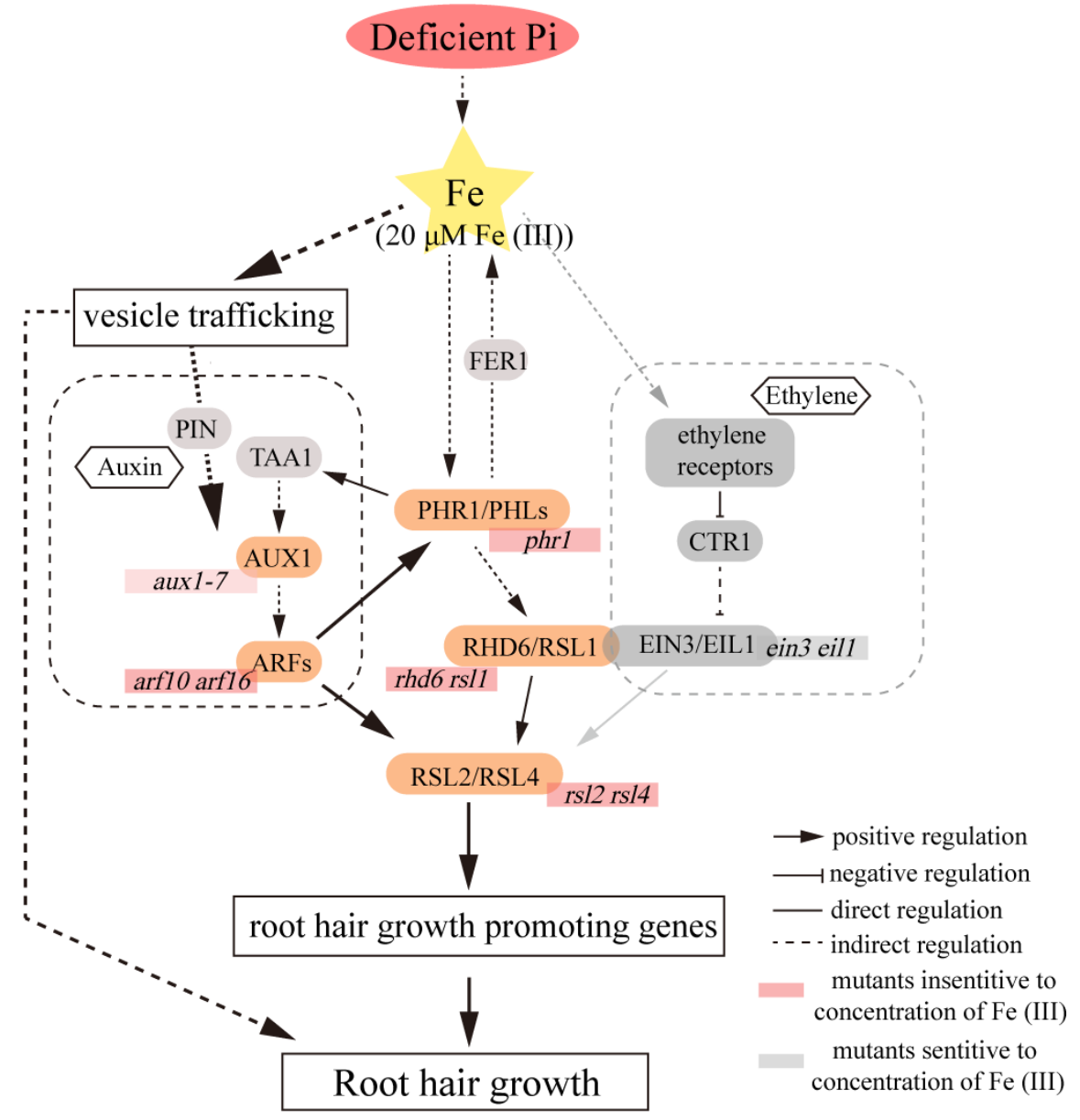

